Differences in Corticospinal Excitability to the Biceps Brachii Between Arm Cycling and Tonic Contraction Are Not Evident at the Immediate Onset of Movement

\author{
By \\ (C) Davis Forman \\ A thesis submitted to the \\ School of Graduate Studies \\ in partial fulfillment of the requirements for the degree of \\ Master of Science in Kinesiology
}

School of Human Kinetics and Recreation

Memorial University of Newfoundland

August, 2015

St. John's

Newfoundland 


\begin{abstract}
With the use of indirect stimulation techniques, it is possible to examine the basic, underlying mechanisms involved with the neuronal control of voluntary motor outputs in humans. By developing a better understanding of how the central nervous system functions during these outputs, we allow for the possibility of improving current rehabilitative and therapeutic strategies for people with neurological injuries and/or diseases. Recently, this type of work has demonstrated that corticospinal excitability is not task-dependent prior to the initiation of a motor output. However, substantial evidence has shown that corticospinal excitability is task-dependent during motor outputs. Considering these two findings, it is highly plausible that a transition occurs in corticospinal excitability from task-independent to task-dependent as movement progresses from rest to steady-state. The timeline of this transition is poorly understood. Therefore, the purpose of this study was examine the possible task-dependent transition in corticospinal excitability from rest to steady-state arm cycling.
\end{abstract}




\section{ACKNOWLEDGEMENTS}

First and foremost, I would like to thank my supervisor and mentor, Dr. Kevin Power for offering me such an incredible opportunity to pursue my master's degree here at Memorial University. Under his guidance, I truly believe that I have received an unrivaled education that has placed me on a path to success for many years to come. I am incredibly grateful for the effort he has put into improving my research experience and I cannot thank him enough for the hundreds of hours that he has selflessly devoted to enriching my young, academic career. I have thoroughly enjoyed the time spent working by his side and I look forward to future collaborations.

I would also like to express my sincere gratitude to my immediate family for their unwavering support during the course of my master's degree. While it has ultimately been an unforgettable experience, there were a few challenges and rough patches along the way; all of which were made easier with you in my corner. To my parents, Bonnie and Steve, thank you so much for this once-in-a-lifetime opportunity to pursue my education away from home. It wouldn't have been possible without you both. And to my siblings, Garrick and Sidney, thank you for always having my back. Your support has helped me in more ways than you realize.

These acknowledgements would not be complete if I were to leave out my fellow co-authors on this project; Devin Philpott and Duane Button. This study, while technically the most difficult I have undertaken to date, was made much easier with Devin's assistance. His reliability in the lab was unimaginably valuable and I would like to thank him for his commitment to this project. And to Duane, thank you for your honest and helpful advice throughout every stage of this thesis. Your unique perspective has 
greatly improved the quality of the research that I have been involved with and I am sincerely grateful for it. 


\section{Table of Contents}

1.1 List of Figures...................................................................................................... vii

1.2 List of Abbreviations .......................................................................................... viii

Chapter 2 Review of Literature …………………………………………………….... 2-1

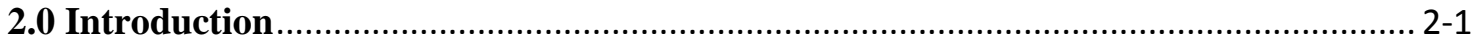

2.01 The Corticospinal Tract …………………………………………………. 2-3

2.02 Assessing Corticospinal Excitability ………………………………………….... 2-5

2.03 Transcranial Magnetic Stimulation .................................................................. 2-7

2.04 Transmastoid Electrical Stimulation............................................................... 2-8

2.05 Examining the Neural Control of Rhythmic Motor Outputs.................................. 2-10

2.06 Pre-Movement Modulation of Corticospinal Excitability for CPG Mediated Motor

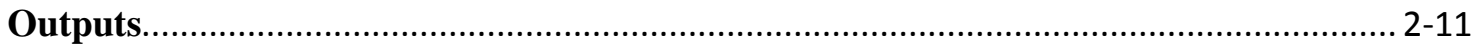

2.07 Corticospinal Modulation During CPG Mediated Motor Outputs .......................... 2-13

2.08 Rhythmic Motor Outputs in Animal Models....................................................... 2-16

2.09 The Mesencephalic Locomotor Region and Movement Initiation........................... 2-18

2.10 Initiation of Rhythmic Motor Outputs in Humans ........................................... 2-20

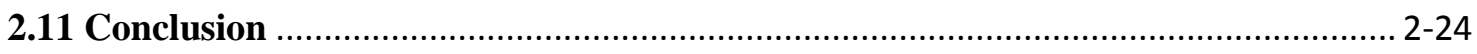

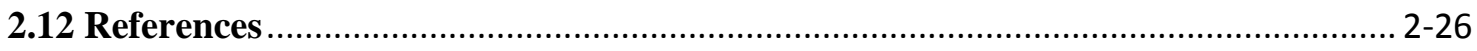

Chapter 3 Differences in Corticospinal Excitability to the Biceps Brachii Between Arm

Cycling and Tonic Contraction Are Not Evident at the Immediate Onset of Movement .... 3-1

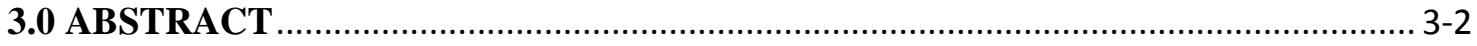

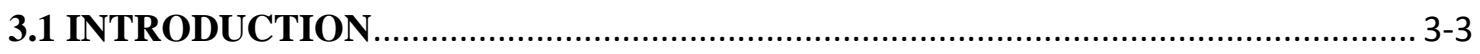

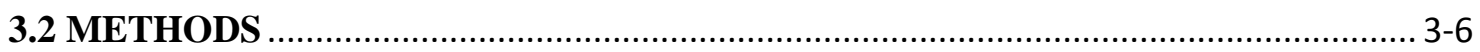

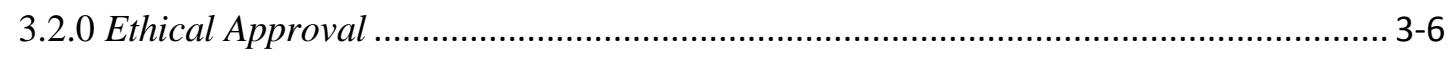

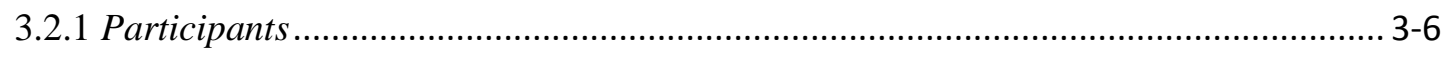

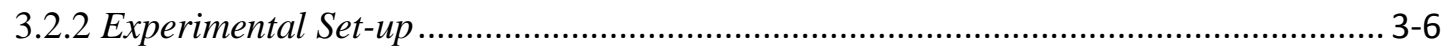

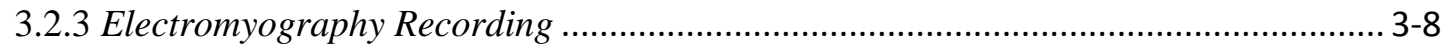

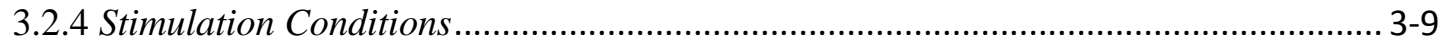

3.2.5 Brachial Plexus Stimulation............................................................................ 3-10 


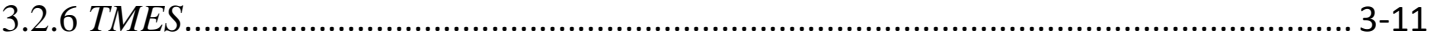

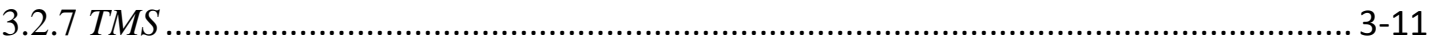

3.2.8 Experimental Protocol ...................................................................................... 3-12

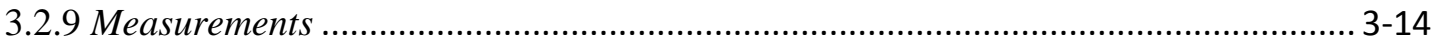

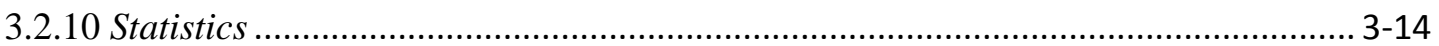

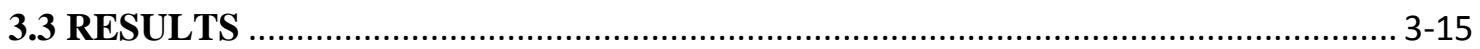

3.3.1 Matched Stimulations.......................................................................................... 3-15

3.3.2 Corticospinal Excitability ................................................................................. 3-15

3.3.3 Spinal Excitability .............................................................................................. 3-17

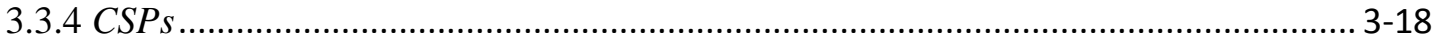

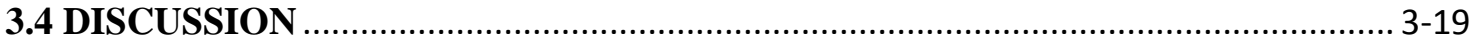

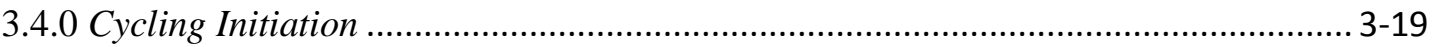

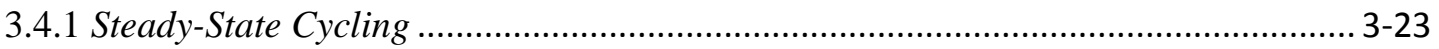

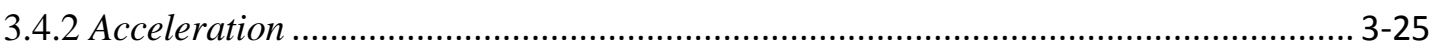

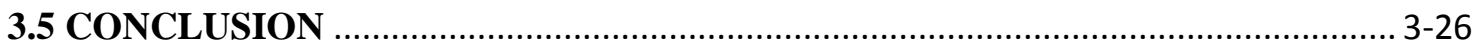

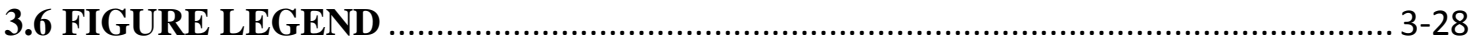

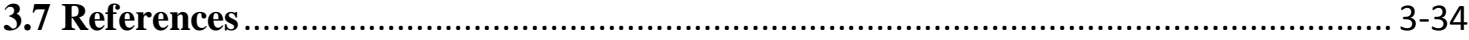




\subsection{List of Figures}

Figure 1 Arm Cyling EMG - Matched MEPs/CMEPs ......................................................... 3-29

Figure 2 Representative MEP Amplitudes.......................................................................... 3-30

Figure 3 Group MEP Amplitudes/Pre-Stimulus EMG …..................................................... 3-31

Figure 4 Group CMEP Amplitudes/Pre-Stimulus EMG .................................................... 3-32

Figure 5 Group CSP Durations ................................................................................ 3-33 


\subsection{List of Abbreviations}

$\% \mathrm{MSO}$ - percent of maximum stimulator output $\mu \mathrm{s}-$ microseconds

AHP - afterhyperpolarization

$\mathrm{cm}$ - centimeter

CMEP - cervicomedullary evoked potential

CNS - central nervous system

$\mathrm{CPG}$ - central pattern generator

CSEP - Canadian society for exercise physiology

CSP - corticospinal silent period

DF - dorsiflexion

D-wave - direct wave

ECR - extensor carpi radialis

EMG - electromyography

EPSP - excitatory post-synaptic potential

FCR - flexor carpi radialis

$\mathrm{Hz}$ - hertz (frequency)

I-wave - indirect wave

$\mathrm{kg}$ - kilograms

$\mathrm{mA}$ - milliamps

MEP - motor evoked potential

MLR - mesencephalic locomotor region

$\mathrm{M}_{\max }$ - maximum $\mathrm{M}$-wave

$\mathrm{ms}$ - milliseconds

$\mathrm{mV}-$ millivolts

MVC - maximal voluntary contraction

MVE - maximal voluntary EMG

M-wave - compound muscle action potential

PD - Parkinson's disease

$\mathrm{PP}$ - peak power

RMS - root mean square

RPM - revolutions per minute

$\mathrm{SD}$ - standard deviation

SE - standard error

TA - tibialis anterior

TMES - transmastoid electrical stimulation

TMS - transcranial magnetic stimulation

$\mathrm{V} / \mathrm{s}$ - volts per second

$\mathrm{VL}$ - vastus lateralis

$\mathrm{V}_{\mathrm{th}}-$ voltage threshold 


\section{Chapter 2 Review of Literature}

\subsection{Introduction}

For most healthy people, walking is viewed as a simple motor task that can be performed with minimal conscious effort. It is, after all, a movement that is mastered as a child. But in reality, walking, like all rhythmic motor outputs, is quite complex. It requires contributions from multiple muscles of the lower body, timely, coordinated movement of both legs, and constant balance as the body sways from step to step. The neural control for all of these parameters is commonly thought to arise solely from the brain, with the spinal cord acting as nothing more than a two-way messenger system that connects the brain to target muscles. We have known this to be untrue as far back as the late $19^{\text {th }}$ century.

Sir Charles Sherrington, who won the Nobel Prize in Physiology or Medicine in 1932 for his work on "...discoveries regarding the function of neurones," was one of the very first pioneers to demonstrate the intricate activity of spinal neurones. His research was fundamental to our understanding of many spinal processes and his work led to several ground-breaking discoveries. One of these discoveries was that muscles with opposing functions (referred to as 'antagonists'; meaning that when they contract, they move a joint in opposite directions) communicate with each other through spinal pathways. This communication is known today as reciprocal inhibition, and functions as such: when a muscle contracts, its antagonists are inhibited and become less likely to produce meaningful force. Future research would demonstrate that reciprocal inhibition is just one of many spinally generated reflexes that play an influential role in movement. 
But spinal contributions to movement, specifically rhythmic movement, do not begin and end with reflexes alone.

Work in animal models has demonstrated that, when void of both cortical and sensory input, it is still possible for rhythmic motor outputs to be generated. This finding has led to the conclusion that specific spinal circuits with the ability to produce the basic characteristics of rhythmic movement must therefore exist (Grillner 1981; Jordan 1998). These circuits are now referred to as central pattern generators (CPGs) and have been discovered in multiple animal species. CPGs function via the phasic activation of antagonist muscles through spinally mediated reciprocal inhibition, thus producing an alternating motor output. An example of this can be seen in the hip flexors/extensors during walking (Dimitrijevic et al. 1998). While the spinal motoneurones to the hip flexors are active in the swing phase of walking, the CPG reciprocally inhibits the spinal motoneurones to the hip extensors. As the foot makes contact with the ground and the leg transitions to the stance phase, inhibition is removed from the hip extensor motoneurones and applied to the hip flexors. This simple, yet essential mechanism creates a rhythm of activation between opposing muscles about a joint, allowing for rhythmic movements to be produced.

There is evidence that rhythmic movements in humans are also produced in part by spinal CPGs (Capaday et al. 1999; Zehr and Stein, 1999; Pyndt and Nielsen, 2003; Carroll et al. 2006; Zehr et al. 2009). A great deal of this research comes from indirect measures of corticospinal tract excitability, which is one of the major descending pathways of the central nervous system (CNS) responsible for voluntary control of movement. Included in this body of work are two of our previous studies, which have 
assessed the excitability of the corticospinal tract between two different motor tasks; arm cycling and a tonic contraction of the elbow flexors. Comparing responses during rhythmic tasks to responses elicited during non-rhythmic tasks is a common method used in this field. As a tonic contraction is not a CPG-mediated output, any discrepancies observed between the two tasks are likely due to the unique control patterns used to modulate rhythmic movements. Using this strategy, we have shown that corticospinal excitability prior to movement is not different between arm cycling and a tonic contraction (Copithorne et al. 2014). During movement, however, corticospinal excitability is elevated while arm cycling (Forman et al. 2014). These two findings suggest that the neural control prior to movement is not influenced by the forthcoming output, but becomes task-dependent during movement. It can thus be inferred that a transition in the modulation of corticospinal excitability from being task-independent to task-dependent must occur once the movement begins.

The time-course of this transition is currently unknown as studies in this line of work typically take measurements once rhythmic movement has reached a constant cadence (i.e. long after the movement began). Such a gap in knowledge is vitally important as this field of study has the potential to benefit therapeutic research involved with CNS disorders. The aim of this review is to discuss the current literature in regards to the modulation of the CNS during voluntary movement.

\subsection{The Corticospinal Tract}

One of the most influential descending pathways involved in the control of voluntary movement is the corticospinal tract. As its name suggests, this pathway 
originates in the motor cortex and synapses onto spinal neurones throughout the length of the spinal cord. Specifically, the tract originates via pyramidal cells located in the $5^{\text {th }}$ layer of the motor cortex. The name 'pyramidal' arises from the pyramid-like, white matter structures of the medulla; the site at which the axons of the corticospinal tract pass through before they decussate, or cross over (Nathan and Smith 1955). These cells have several names, but are usually referred to as upper motoneurones in a more general sense. The reason being is that they are located within the brain and are thus anatomically higher than their spinal motoneurone counterparts. In this review, pyramidal cells will be written as upper motoneurones. The location of upper motoneurones within the cortex varies among individuals, but more than $60 \%$ of these cells are usually found within the supplementary, premotor and primary motor areas of the frontal lobe (Dum and Strick 2009; Jane et al. 1967). Additional cells have been observed in the primary sensory area and the parietal cortex (Lemon 2008). The axons of the corticospinal tract take a relatively linear path to the brainstem, passing through several structures of the cerebral cortex and the midbrain on the way. Here, they form the pyramids of the medulla, which occurs just rostral to the point where the tract decussates (Nathan and Smith 1955). The exact percentage of the tract that decussates varies between reports, but a common estimate is that $80-90 \%$ of the corticospinal tract axons cross over to the contralateral side at the medulla (Kaneko et al. 1997). At this point, the corticospinal tract branches into two separate pathways with $80-90 \%$ of axons that crossed over forming the lateral corticospinal tract and the remaining axons forming the anterior corticospinal tract. Axons of both tracts then descend down the spinal cord to synapse onto specific spinal neurones. The axons of the lateral corticospinal tract synapse directly onto spinal neurones located 
on the ipsilateral side in which they travelled (contralateral to their origin within the motor cortex). The axons of the anterior corticospinal tract, once they have reached their appropriate spinal level, must first decussate to the contralateral side before they synapse onto spinal neurones (Nathan et al. 1990; Eyre 2003). This means that while the two tracts do not travel along the same pathway, they both innervate the contralateral side of the body from which they originated. Upper motoneurones travelling along both tracts will synapse onto either a spinal interneurone or directly onto a spinal motoneurone. The exact percentage that make monosynaptic connections with spinal motoneurones varies between muscles. For the biceps brachii, it is believed that they possess a large monosynaptic component (Petersen et al. 2002; Ugawa Y et al. 1991). The spinal motoneurones, which are located in the anterior horn of the grey matter at their respective spinal level, then exit the spinal cord through a ventral root. For the biceps brachii and most of the muscles of the upper limb, these axons exiting the spinal cord eventually form part of the brachial plexus. Specifically, the brachial plexus is formed from the ventral roots exiting the spinal cord at C5-C8 and T1 (Johnson et al. 2006). The brachial plexus then branches off into many individual nerves that innervate the muscles of the shoulder, the arm and the hand. One of these nerves is the musculocutaneous nerve, which innervates the muscle fibers of the long and short heads of the biceps brachii.

\subsection{Assessing Corticospinal Excitability}

The electrical properties of the corticospinal tract are in a constant state of change. At all times, the corticospinal tract receives synaptic input from a wide range of sources, including other regions of the brain, the brain stem, and sensory feedback from the 
peripheral nervous system. The sum of this input, as well as any changes in intrinsic electrical properties of the cells that compose this pathway, modulates the responsiveness of the corticospinal tract making it more or less likely to produce a motor output. In neurophysiology, this responsiveness is referred to as excitability. For example, if an individual is at rest, their body is completely relaxed and they are not consciously thinking about movement, corticospinal excitability is likely low. If, however, they were to move their limbs, contract a muscle, or even just think about moving, corticospinal excitability would increase, meaning that the pathway has become more responsive. In humans, the excitability of the corticospinal tract is typically measured indirectly using either electrical or magnetic stimulation techniques. These methods apply stimulation over a specific region of the pathway, such as the motor cortex, brainstem or spine, and measure the evoked response in a target muscle (McNeil et al. 2013). The size of the response, or how quickly the response occurred, can indicate the excitability of the corticospinal tract at the time the stimulation was delivered. Using the example mentioned above, if a stimulation of ' $\mathrm{X}$ ' intensity was delivered to the motor cortex while a participant was at rest, it is possible that no response would be observed due to the low excitability of the corticospinal tract. Under different conditions, such as a mild muscle contraction, that same stimulation intensity (i.e. ' $\mathrm{X}$ ') could result in a very large response as the excitability of the pathway would be much higher; neurones along the corticospinal tract made to fire action potentials more easily.

While many different techniques exist to measure corticospinal excitability in humans, each with their own strengths and limitations, this review will be focused on 
two. Specifically, transcranial magnetic stimulation (TMS) and transmastoid electrical stimulation (TMES) are discussed in greater detail below.

\subsection{Transcranial Magnetic Stimulation}

TMS is a technique that activates the motor cortex by inducing a rapid change in the magnetic field, or magnetic pulse, over the skull (Barker et al. 1985). Typically, this technique does not directly activate the upper motoneurones of the corticospinal tract as they are located deep within the motor cortex. Instead, TMS activates interneurones in the upper layers of the motor cortex, which then synapse onto the corticospinal tract neurones. This is referred to as trans-synaptic activation. The number of synapses that occur before the signal reaches the corticospinal tract depends on which of the individual cells in the upper layers of the motor cortex are being activated. Therefore, the sum of the synaptic input delivered to the upper motoneurones does not necessarily arrive at the same time (i.e. temporal summation occurs). Consequently, TMS results in the production of multiple descending volleys rather than just one, synchronized, evoked response. This has previously been demonstrated in practice as it is possible to observe these volleys at the spinal cord via epidural electrodes (Burke et al. 1993). These are known in literature as indirect waves (I-waves) and are the result of the trans-synaptic nature of TMS (Di Lazzaro et al. 1998). It is possible for certain individuals, and at high enough stimulation intensities, to directly stimulate the corticospinal tract. These are referred to as direct waves (D-waves) and can be distinguished from I-waves in electromyography recordings (EMG) by their shortened latency of approximately 1-1.4 ms (Burke et al. 1993; Di Lazzaro et al. 1998). Responses elicited from TMS are usually recorded from a target 
muscle as a compound muscle action potential; termed a motor evoked potential (MEP) (Taylor et al. 2002). The size of the peak-to-peak amplitude, the onset latency of the evoked response, and the corticospinal silent period (CSP) (the duration of time from the onset of the stimulus artifact to when voluntary EMG returns following the evoked response) of the MEP are used to assess changes in corticospinal excitability under different experimental conditions. However, an important point to remember is that the corticospinal tract is composed of cortical and spinal components. It is therefore possible that differences in excitability (i.e. changes in the MEPs) between experimental conditions can be due to changes at the supraspinal level, the spinal level, or both. But determining the source(s) of that change is difficult with TMS alone as MEPs are an indicator of overall corticospinal excitability. Due to this limitation, TMS is often used alongside an independent measure of spinal excitability in order to interpret MEPs to a greater degree. One such technique is transmastoid electrical stimulation (TMES)

\subsection{Transmastoid Electrical Stimulation}

While not as common as some other methods, TMES is a technique that involves an electric current being passed through surface electrodes placed on the mastoid processes. Stimulation at this location activates the axons of the corticospinal tract (axons of the upper motoneurones) near the cervicomedullary junction (Taylor 2006). This site has been determined as an optimal location as the corticospinal tract decussates at this level and the bending of the axons provides a larger, and more easily activated surface area (Amassian et al. 1992; Maccabee et al. 1993). The resulting effect is a single descending volley in the muscles of the upper limb, and in some individuals, the lower 
limb (Ugawa et al. 1991; Ugawa et al. 1995). For the upper limb, the single volley produced by TMES proceeds down the axons of the upper motoneurones, synapses with spinal motoneurones, and is then recorded at a target muscle. This evoked response is known as a cervicomedullary evoked potential (CMEP) and is used as a means to distinguish changes in spinal excitability under different experimental conditions (Taylor 2006). It is therefore an appropriate choice to be used alongside TMS in order to differentiate changes in corticospinal excitability as being derived from either supraspinal or spinal sources.

An issue worth noting with TMES is that stimulation between the mastoids has a tendency to activate the ventral roots emerging from the spinal cord, along with the desired corticospinal tract (Ugawa et al. 1991). Ventral roots have been observed to bend upon their exit from the spinal cord, which provides a second, easily activated site following electrical stimulation (Rossini et al. 1985; Mills and Murray, 1986). If the ventral roots are indeed activated, this will likely appear in an EMG trace as a sudden decrease in the CMEP onset latency by approximately 2 ms (Ugawa et al. 1991). This can be a potential problem, as activation of the axons of the spinal motoneurones no longer represents the excitability of the motoneurone itself. CMEP onset latency must therefore be carefully monitored when using TMES as an experimental technique.

One assumption that must be met when using different techniques to assess corticospinal and spinal excitability is that both techniques activate similar axons within the corticospinal tract. Paired TMS and TMES stimulation paradigms have demonstrated that these two techniques do indeed activate similar motor pools, as the antidromic action potentials produced by TMES appear to collide with and cancel the descending volleys 
induced by TMS (Taylor et al. 2002). This finding suggests that both techniques travel along the same pathways, thus validating the use of TMS and TMES to examine corticospinal excitability.

\subsection{Examining the Neural Control of Rhythmic Motor Outputs}

In both animal models and human research, an effective method of establishing the task-specific mechanisms involved in the production of rhythmic movement is by comparing a rhythmic motor output to a non-rhythmic motor output. For the type of indirect research that is common in human work, this is most often conducted by contrasting a rhythmic motor output (such as walking or cycling) with a tonic, or isometric contraction. This type of methodology has been previously used in both leg (Pyndt et al. 2003) and arm cycling (Carroll et al. 2006; Forman et al. 2014). The reason for this strategy's effectiveness is that the basic, alternating characteristics of rhythmic motor outputs, such as leg and arm cycling, are generated in part by spinal pathways (Zehr et al. 2004), which are absent during tonic contractions. Tonic contractions can therefore be used as a control of sorts, so long as the overall output between tasks is the same. If that assumption is met, and the intensity of the two motor outputs is equal (the level of muscle activity, or EMG, is matched between tasks), any differences in corticospinal excitability may be due to differences in the central control of the two tasks. In that scenario, the influence of CPGs on the rhythmic motor output becomes a possible explanation for the observations. 


\subsection{Pre-Movement Modulation of Corticospinal Excitability for CPG Mediated Motor Outputs}

The modulation of corticospinal excitability during rhythmic motor outputs is complex, but this pattern of excitability begins before movement actually starts. Power and colleagues (Power and Copithorne, 2013) demonstrated that corticospinal excitability to the biceps brachii was enhanced $50 \mathrm{~ms}$ prior to the onset of voluntary EMG from the initiation of an arm cycling task. Corticospinal and spinal excitability were assessed through the use of single pulse TMS and TMES, respectively. Both of these stimulations were delivered approximately 50 ms before the expected onset of biceps brachii EMG. This point of delivery varied between individuals and was found by measuring the reaction time of each participant. Once reaction time was determined, the stimulations were programmed to arrive automatically $50 \mathrm{~ms}$ beforehand. Measurements were then taken during two separate experimental conditions; arm cycling and rest. For arm cycling, participants were given an auditory warning signal that instructed them when to begin cycling. For the rest condition, which acted as a control, participants were instructed not to move or think of moving upon hearing the auditory cue. An increase in the peak-topeak amplitude of the MEP, as well as a decrease in the MEP onset latency, was observed prior to arm cycling when compared to rest. Both CMEP amplitude and the CMEP onset latency were not different between experimental conditions. These results suggest that the enhancement in corticospinal excitability prior to arm cycling is likely supraspinally mediated as there was no change in spinal excitability. It has been suggested that the increase in supraspinal excitability may result from increases in intracortical facilitation (Nikolova et al. 2006), or from a progressive decrease in interhemispheric inhibition 
(Duque et al. 2007). It is also likely that, based on the reduced onset latency of the MEP prior to arm cycling, fewer successive I-waves may have been needed to produce said MEP. If cortical motoneurones were excited prior to movement, and firing thresholds were hyperpolarized, then it is possible that the development of MEPs would not have been dependent on the temporal summation of excitatory post-synaptic potentials (EPSPs).

It was proposed that a possible reason for the lack of modulation in spinal excitability in the aforementioned study (Power and Copithorne, 2013) was that the measurements were not taken close enough to the onset of muscle activity. The case may have been that spinal changes were indeed occurring before movement, but simply not 50 ms beforehand. Reasoning for this line of thought arose from findings in the soleus, whereby CMEPs have been shown to increase $25 \mathrm{~ms}$ prior to dorsiflexion (Geertsen et al. 2010). However, in a follow-up study on the biceps brachii, Copithorne et al. (2014) discovered that this is likely not the case, as similar results were found at $25 \mathrm{~ms}$ prior to arm cycling. As with their earlier research, TMS and TMES were used to assess corticospinal and spinal excitability, respectively, of the biceps brachii. Both stimulation techniques were elicited approximately $25 \mathrm{~ms}$ before the onset of EMG induced from the initiation of arm cycling. The peak-to-peak amplitude of the MEP was found to increase and the onset latency was shown to decrease prior to arm cycling when compared with rest. Both CMEP amplitude and onset latency were unchanged. A methodological difference with their previous work is that Copithorne et al. (2014) also compared precycling measures with pre-tonic measures. Following cycling trials, participants were required to produce a position and intensity matched (same EMG between motor tasks) 
tonic contraction following an auditory cue. As with cycling, TMS and TMES were placed approximately $25 \mathrm{~ms}$ prior to the onset of EMG produced by the tonic contraction. An increase in MEP amplitude was found to coincide with a decrease in the onset latency when compared with rest. Neither CMEP amplitudes nor onset latencies were shown to change. The most interesting finding, however, was that neither the MEP amplitude nor the MEP onset latency were significantly different between the two motor tasks.

According to these findings, it appears that corticospinal excitability to the biceps brachii increases prior to arm cycling. Evidence suggests that this is due to changes in supraspinal excitability, with variations in intracortical facilitation and interhemispheric inhibition as possible mechanisms. It also appears that spinal excitability remains unchanged, at both 50 and $25 \mathrm{~ms}$ prior to movement. But perhaps the most important point from this research is that this pattern of corticospinal modulation prior to arm cycling does not appear to be different than that prior to an intensity matched tonic contraction. This finding suggests that pre-movement modulation of supraspinal mechanisms may be uniform across motor outputs (i.e. not task-dependent).

\subsection{Corticospinal Modulation During CPG Mediated Motor Outputs}

The production of a rhythmic motor output like arm cycling is accomplished through a complex combination of descending drive from supraspinal factors, reorganization of intrinsic spinal excitability, and reflexive adjustments via peripheral pathways. However, while their modulation is notably intricate, these components function together in a cooperative manner. During leg cycling, Sidhu et al. (2012) demonstrated that corticospinal and spinal excitability to lower limb muscles are 
modulated throughout the phase of a full cycle. They used TMS and TMES to preferentially activate the corticospinal tracts to the vastus lateralis (VL), and elicited these techniques throughout a full revolution of leg cycling. Responses were then grouped into 12 'bins' according to the time in which they were delivered. Each of the 12 'bins' corresponded to one of the 12 pedal positions relative to the face of a clock. It was discovered that, when normalized to background EMG, MEP amplitudes of the VL were largest just prior to the most active phase of the muscle, and were significantly smaller from that point on. CMEP amplitudes were found to follow a similar pattern of modulation. This led to the conclusion that the contribution of the motor cortex is enhanced prior to maximum muscle activation, but spinal-factors are largely responsible for phase-dependent modulation of corticospinal excitability. Similar conclusions have been drawn in the flexor carpi radialis (FCR) during arm cycling. Carroll et al. (2006) used TMS to assess corticospinal excitability and electrically induced H-reflexes to assess spinal excitability to the FCR at four different positions throughout a cycling revolution (3, 6, 9 and 12 o'clock relative to a clock face). These techniques were also delivered during position and intensity matched (equal EMG) tonic contractions. Both MEP amplitude and the H-reflex during a tonic contraction were significantly larger at the 6 o'clock position when compared to arm cycling. H-reflexes were also larger during tonic trials at the 3 o'clock position. Additionally, Zehr and colleagues (Carroll et al. 2006) discovered that sub-threshold TMS significantly facilitated the H-reflex during tonic contractions, but had no influence while cycling. This data suggests that the responsiveness of the motor cortex is depressed during an arm cycling task, and that spinal contributions likely make up a larger portion of the central control. The findings of 
a more recent paper suggest that not only is corticospinal excitability phase- and taskdependent, but likely muscle-dependent as well. Forman et al. (2014) examined corticospinal excitability to the biceps brachii during arm cycling through the use of both TMS and TMES. Stimulations were elicited automatically at pedal positions 3, 6 and 12 relative to a clock face. As with Zehr and colleagues (Carroll et al. 2006), evoked responses during cycling trials were then compared with position and intensity matched tonic contractions. Similar to previous research, both MEPs and CMEPs were found to be modulated throughout a full cycle. But unlike in the FCR (Carroll et al. 2006), MEPs elicited in the biceps brachii at the 3 and 6 o'clock position were significantly larger during arm cycling when compared with a tonic contraction. CMEPs were also larger at 3 o'clock during arm cycling, which may be fully or partially responsible for the increase in the MEP at the same position. One explanation for this observed discrepancy is that the roles of the FCR and biceps brachii during arm cycling are remarkably different. FCR activity is produced mainly to stabilize the wrist, which means it is continuously active during this motor output. It demonstrates very little phasic activation, which is a striking contrast to the biceps brachii. As one of the prime movers during arm cycling, the biceps brachii produce large bursts of EMG during elbow flexion (3 to 9 o'clock) and very little EMG activity during elbow extension (9 to 3 o'clock). It can be surmised that the role of a muscle during a cycling motor output heavily influences the manner in which corticospinal excitability is modulated. However, regardless of the inter-muscle differences that appear to be present, it is clear that corticospinal excitability during arm cycling for both the FCR and the biceps brachii is generated differently than during a 
tonic contraction. This may be partially due to the contributions of CPGs during arm cycling, which would be absent for a tonic contraction.

\subsection{Rhythmic Motor Outputs in Animal Models}

While human research into the neural mechanisms of rhythmic motor outputs has added fundamental knowledge to the field, there is a great deal of information that must still be derived from animal models. This is largely due to the limitations of indirect neurophysiological assessment techniques associated with human research. Of course, that is not to say that animal models are not without their own limitations, but using this form of research, it is possible to examine the CNS at the cellular level. This work has demonstrated that while descending input is required to initiate locomotor outputs (Jordan et al. 2008), the activity itself is modulated by the alternating activity of spinal CPGs (Grillner 1981; Jordan 1998). Additionally, spinal motoneurones, which are not directly assessed in human research, have been found to undergo substantial intrinsic changes to their electrical properties prior to and throughout locomotor outputs. Intracellular recordings have previously been made in the decerebrate cat during rest, as well as fictive locomotion. It was found that the voltage threshold $\left(\mathrm{V}_{\text {th }}\right)$ (the voltage that must be obtained in order for an action potential to fire) is hyperpolarized by an average of $-8 \mathrm{mV}$ during fictive locomotion (Krawitz et al. 2001), meaning that the spinal motoneurone requires substantially less synaptic input to reach $V_{\text {th }}$. It has also been shown in the decerebrate cat that the amplitude of the afterhyperpolarization (AHP) (period following an action potential where the membrane potential falls below resting levels) is reduced during locomotor outputs (Brownstone et al. 1992), meaning that subsequent action 
potentials can occur at a higher firing frequency. Similar findings have been observed during fictive locomotion in rat models (Schmidt, 1994). Lastly, the excitatory component of the locomotor drive potential (the alternation of excitation/inhibition by spinal CPGs) appears to respond in a voltage-dependent manner (Brownstone et al. 1994). As the membrane potential of the motoneurone depolarizes, motoneurone excitability is amplified. These mechanisms appear to function in a cooperative manner in order to increase the excitability of spinal motoneurones during locomotion. However, as was stated in an earlier section, a limitation with this kind of research is that, while it sheds light on the modulation of spinal pathways during rhythmic motor outputs, it does not demonstrate whether or not this modulation is task-dependent. Are the changes in spinal motoneurone properties that occur during locomotor outputs similar for all motor outputs? This question was examined in the adult decerebrate cat by Power and colleagues (Power et al. 2010). In this study, $\mathrm{V}_{\text {th }}$ was measured using intracellular recordings from semimembranosous motoneurones during fictive scratch and fictive weight support. $\mathrm{V}_{\text {th }}$ was measured from spikes under two different experimental conditions: 1) following intracellular current injection which elicited action potentials, and 2) action potentials occurring spontaneously during the depolarizing phase of fictive scratch or weight support. $\mathrm{V}_{\text {th }}$ was defined as the point at which the membrane potential rapidly depolarized at a rate of $\geq 10 \mathrm{~V} / \mathrm{s}$. Fictive scratch of the ipsilateral hind limb was achieved by manual stimulation of the ipsilateral ear. For fictive scratch, measurements of $\mathrm{V}_{\text {th }}$ were taken before, during and after a short bout ( $\sim 7$ seconds). On average, the $V_{\text {th }}$ during fictive scratch was hyperpolarized by $1.8 \mathrm{mV}$ compared to rest. This value recovered to resting levels within 6 seconds following the cessation of fictive scratch. 
These findings support earlier research during fictive locomotion, suggesting that similar motoneurone excitability modulation occurs between rhythmic motor outputs. In order to establish that this modulation was unique to rhythmic motor outputs, $\mathrm{V}_{\text {th }}$ was the assessed during fictive weight support. To rule out variability of $\mathrm{V}_{\text {th }}$ between motoneurones, the same motoneurones assessed during fictive scratch were also assessed during fictive weight support. This was achieved by manually stimulating the contralateral ear, thus producing fictive scratch in the contralateral hind limb. Fictive scratch of the contralateral limb results in the activation of ipsilateral extensor motoneurones of the hind limb to produce a tonic contraction of the ipsilateral limb, referred to as fictive weight support (Perreault et al. 1999). Measurements were again taken before, during and after a short bout of fictive weight support. On average, the $\mathrm{V}_{\text {th }}$ during fictive weight support was depolarized by $3.7 \mathrm{mV}$ when compared with rest, which contrasts the findings during fictive scratch. From these findings, intrinsic excitability of spinal motoneurones appears to increase during fictive scratch, but decrease during fictive weight support. This suggests that changes in motoneurone excitability are task-dependent. It also highlights the importance of comparing measures of the CNS between tasks, both in animal models and human research.

\subsection{The Mesencephalic Locomotor Region and Movement Initiation}

While it is widely accepted that spinal mechanisms provide the largest contribution to the characteristic, alternating activation of antagonist motoneurones during locomotor outputs, these pathways require supraspinal input in order to become active. This discovery was made in 1966 when researchers working with decerebrate cats 
elicited locomotion by applying stimulation to a specific site in the midbrain (Shik et al. 1966). According to these authors, "The cat which before stimulation was immobile suddenly "came to life," stood up on its legs and began to move setting in motion or accelerating the treadmill belt... It is assumed that the control of walking and running in the intact animal is brought about through change in the excitability of a definite region of the mid-brain," (Shik et al. 1966). Subsequent research would eventually lead to this region being termed the mesencephalic locomotor region (MLR). Specifically, the MLR is located in the mesopontine reticular formation of the brainstem (Skinner et al. 1990), and possesses cells that directly synapse onto cells of the reticulospinal tract (Grillner, 1981; Jordan, 1991; Rossignol, 1996; Grillner et al. 1997; Jordan, 1998; Whelan, 1996). As with the corticospinal tract, the reticulospinal tract is one of the major, descending pathways in the CNS that contributes to voluntary movement. However, while a large portion of the upper motoneurones in the corticospinal tract terminate via synaptic connections with spinal motoneurones, reticulospinal neurones project onto spinal interneurones that form CPGs (Grillner et al. 1997; Jordan, 1998). Thus, excitation of the MLR indirectly produces locomotor outputs via activation of the reticulospinal pathway. However, while the MLR is considered to be the region with the largest concentration of neurones devoted to the initiation of locomotion, it is not the only structure with projections to the reticulospinal pathway. The lateral hypothalamus and the cerebellum provide direct, excitatory input to the reticulospinal tract, while the medial hypothalamus delivers indirect, excitatory input through the MLR (Grillner et al. 1997). Additionally, several structures that form the basal ganglia have been shown to control the MLR through inhibitory input, in which excitation of these pathways would cease the activity 
of the MLR (See Fig. 1 in Jordan, 1998 for summary of main structures). This multitude of descending pathways involved in locomotor initiation form a redundancy that may appear unnecessary, but consequently provides the possibility of locomotor recovery following an injury or neurodegenerative disease.

\subsection{Initiation of Rhythmic Motor Outputs in Humans}

At present, no study has characterized how corticospinal excitability is modulated at the immediate initiation of a cycling task. There have, however, been studies to examine this pathway during gait initiation. Considering gait is a rhythmic motor output, and likely shares many mechanical and neural control properties with cycling, the findings of this research may have relevance to arm cycling. Hiraoka and Abe (2007) examined the influence of gait initiation in healthy individuals on corticospinal excitability to the ankle flexors and extensors. TMS was used to assess changes in corticospinal excitability and H-reflex was used as a measure of spinal excitability. The "initiation of gait" was defined as the onset of ankle dorsiflexion (DF) that occurred as the participant moved from postural phase (standing still) to swing phase (dominant leg stepping forward). This was assessed by an electrogoniometer. Stimulations were elicited at $180 \mathrm{~ms}$ prior to DF and $150 \mathrm{~ms}$ after DF in $30 \mathrm{~ms}$ intervals (11 total time points). It was found that the soleus H-reflex was depressed prior to movement when compared to rest and up to $30 \mathrm{~ms}$ after the ankle began to DF, which has been confirmed in previous studies (Edamura et al. 1991; Hiraoka et al. 2006). MEPs elicited in the soleus were also depressed prior to DF, but increased significantly once gait was initiated. H-reflexes were not elicited in the tibialis anterior (TA), but MEPs were and these values were larger both 
prior to and after DF when compared to control. The decrease in the soleus MEPs seen prior to movement may be explained by the depression of the soleus H-reflex at the same time point. However, that does not explain the soleus MEPs following DF, which increased despite the soleus H-reflex remaining depressed. This may be a 'spill over' effect from the increase of activity in the lower limb as a whole when gait is initiated. One possible site of this 'spill over' may be from the TA, which demonstrated greater MEPs at the same time points. However, the most noteworthy point from this study was the lack of change in both the soleus H-reflex and the TA MEPs from pre-gait to gait initiation. This finding suggests that patterns of pre-movement corticospinal excitability may persist once movement starts. Whether or not this modulation changes in the later stages of walking once an individual has attained their optimal stride frequency (steadystate) has not been assessed.

A second study conducted by the same research group has characterized corticospinal excitability between the start of a rhythmic and non-rhythmic motor task. Goto et al. (2014) used TMS to assess corticospinal excitability in the soleus and the TA, and the H-reflex to assess spinal excitability in the soleus between a discrete and rhythmic motor task. The discrete (and therefore, non-rhythmic) motor output used in this study was a single, dynamic movement of ankle dorsiflexion followed by ankle plantar flexion. The pace of the entire movement was $1 \mathrm{~Hz}$ and was preceded and followed by a moment of rest (i.e. the movement was not repeated). The rhythmic motor output was simply a repetition of ankle dorsiflexion and plantar flexion with no rest occurring between cycles (10 cycles were completed in total for each trial). This was also kept to a constant pace of $1 \mathrm{~Hz}$ (one round of dorsiflexion and plantar flexion/second) in order to match the speed of 
the two movements. While this type of movement is not a commonly used task within the field, repetitive and periodic flexion and extension of the ankle shares many characteristics with other, well studied rhythmic motor outputs, such walking and cycling. Nevertheless, the 'rhythmicity' of the movement has been brought into question, thus the findings of this work should be interpreted with caution. The stimulations (TMS and Hreflex) were automatically delivered while the ankle was plantar flexing. At this point, the soleus showed signs of muscle activity while the TA was relatively relaxed. For the rhythmic motor output, this position received stimulations during the first cycle of dorsi/plantar flexion as well as the $10^{\text {th }}$ cycle. Due to its nature, the discrete motor task received stimulations in the first and only cycle. It was found that there were no differences in any of the measures taken between discrete ankle movement and the first cycle of rhythmic ankle movement. However, TA MEPs in the $10^{\text {th }}$ cycle of rhythmic ankle movement were significantly larger than MEPs elicited during the $1^{\text {st }}$ cycle, and by extension, discrete ankle movement.

There are a number of important conclusions that can be drawn from these findings. The first is that there appears to be no difference in corticospinal or spinal modulation between the beginning of a rhythmic motor task (or, at the very least, the type of rhythmic movement chosen in this study) and a discrete motor task. Second, and perhaps more importantly, the results of the $10^{\text {th }}$ cycle would indicate that supraspinal excitability is elevated as a rhythmic motor output progresses. It may therefore be possible that the beginning of a rhythmic motor task is under different central control parameters than the later stages of the movement. 
Indirect evidence from Parkinson's disease (PD) research may provide additional support for this conclusion. A common, clinical feature of PD is bradykinesia, which is the slowing of movement. This symptom is characterized by the impairment of an individual's capacity to plan, initiate and execute motor outputs (Berardelli et al. 2001). This often manifests as a difficulty to initiate gait (Gantchev et al. 1996; Rosin et al. 1997). Abnormalities of gait initiation in people with PD has been previously described. Okada et al. (2011) examined several parameters in the first three steps of gait following gait initiation in people with and without PD. Measurements included step length, step width, step time and step velocity. For step length, width and velocity, any differences between people with PD and the healthy controls were consistent throughout steps 1 to 3 . For example, the first step of gait in people with PD was significantly shorter than healthy controls, as was step 2 and step 3. However, it was demonstrated that the duration of the initial step in patients with PD was significantly longer (0.16s) compared to healthy controls. Interestingly, no differences in duration were observed in steps 2 or 3. This suggests that certain motor pathways affected by PD may be specific to the initial moments of the movement, but not the later stages of the rhythmic motor output that follows. Perhaps the most well studied component of these pathways is the basal ganglia, which has been shown to play an important role in the initiation of movement, but is impaired in PD (Flowers 1976; Evarts et al. 1981). These findings suggest that some of the supraspinal pathways affected in people with PD may be modulated differently in the healthy population depending on the phase of the forthcoming movement (initiation vs steady-state). 


\subsection{Conclusion}

Animal research has demonstrated that locomotor outputs are initiated primarily by the MLR, which provides excitatory, synaptic input to the reticulospinal tract and activates spinally located CPGs. During locomotion, the CPG functions to alternatively activate/inhibit motoneurones that innervate opposing muscle groups in a flexion/extension rhythm. While this process requires descending input in order to become active, its function is not dependent on either descending or afferent pathways. Additionally, rhythmic motor outputs result in a reconfiguration of intrinsic electrical properties in spinal motoneurones; increasing their excitability and making them more responsive to synaptic input. It has been demonstrated that this modulation is unique to rhythmic motor tasks as similar results have not been observed during tonic or isometric outputs.

In humans, the central control of voluntary movement is typically measured through the corticospinal tract via indirect stimulation techniques. These methods have revealed that corticospinal excitability is phase, muscle and task specific during rhythmic motor outputs such as leg and arm cycling. However, research regarding pre-movement corticospinal excitability using TMS and TMES has revealed similar modulation patterns prior to arm cycling and prior to intensity-matched, tonic contractions. According to this work, it can be surmised that a progression in the task-specific nature of corticospinal excitability from pre-movement to movement likely occurs at some point once movement begins. The limited research that currently exists indicates that this transition may not be immediate, leaving the possibility that the initial portions of a rhythmic motor output and the later, steady-state phases may be under different neural control mechanisms. 
However, evidence for this statement is presently lacking, and the progression of corticospinal excitability between a cycling task and a non-rhythmic motor output has not been assessed. This is an important gap in our knowledge that requires considerable research in order to gain a better understanding of how humans initiate rhythmic movements. Research of this nature has the potential to provide insight and improve current therapies/treatments for people with neural diseases and/or injuries. 


\subsection{References}

Amassian VE, Eberle L, Maccabee PJ, Cracco RQ. (1992) Modelling magnetic coil excitation of human cerebral cortex with a peripheral nerve immersed in a brain-shaped volume conductor: the significance of fiber bending in excitation. Electroencephalogr Clin Neurophysiol, 85: 291-301

Barker AT, Jalinous R, Freeston IL. (1985) Non-invasive magnetic stimulation of human motor cortex. Lancet, 1: 1106-1107

Berardelli A, Rothwell JC, Thompson PD, Hallett M (2001). Pathophysiology of bradykinesia in Parkinson's disease. Brain, 124:2131-2146

Brownstone RM, Gossard JP, Hultborn H (1994). Voltage-dependent excitation of motoneurones from spinal locomotor centres in the cat. Exp Brain Res, 102:34-44

Brownstone RM, Jordan LM, Kriellaars DJ, Noga BR, Shefchyk SJ (1992). On the regulation of repetitive firing in lumbar motoneurones during fictive locomotion in the cat. Exp Brain Res, 90:441-455

Burke D, Hicks R, Gandevia SC, Stephen J, Woodforth I, Crawford M. (1993) Direct comparison of corticospinal volleys in human subjects to transcranial magnetic and electrical stimulation. $J$ Physiol, 470: 383-393

Capaday C, Lavoie BA, Barbeau H, Schneider C, Bonnard M (1999). Studies on the corticospinal control of human walking. I. Responses to focal transcranial magnetic stimulation of the motor cortex. J Neurophysiol, 81: 129-139

Carroll TJ, Baldwin ER, Collins DF, Zehr EP (2006). Corticospinal excitability is lower during rhythmic arm movement than during tonic contraction. J Neurophysiol, 95: 914921

Copithorne DB, Forman D, Power K (2014). Premovement changes in corticospinal excitability of the biceps brachii are not different between arm cycling and an intensitymatched tonic contraction. Motor control

Di Lazzaro V, Oliviero A, Profice P, Saturno E, Pilato F, Insola A, Mazzone P, Tonali P, Rothwell JC. (1998) Comparison of descending volleys evoked by transcranial magnetic and electric stimulation in conscious humans. Electroencephalography and Clinical Neurophysiology/Electromyography and Motor Control, 109: 397-401 
Dimitrijevic MR, Gerasimenko Y, Pinter MM (1998). Evidence for a spinal central pattern generator in humans. Annals of the New York Academy of Sciences, 860:360-376

Dum RP, Strick PL (2009). The origin on corticospinal projections from the premotor areas in the frontal lobe. J Neurosci 11:667-689

Duque J, Murase N, Celnik P, Hummel F, Harris-Love M, Mazzocchio R, Olivier E, Cohen LG. (2007) Intermanual differences in movement-related interhemispheric inhibition. J Cogn Neurosci, 19: 204-213

Edamura M, Yang JF, Stein RB. (1991) Factors that determine the magnitude and time course of human H-reflexes in locomotion. J Neurosci, 11: 420-427

Evarts EV, Teravainen H, Calne DB (1981). Reaction time in Parkinson's disease. Brain, 104: $167-186$

Eyre J (2003). Development and plasticity of the corticospinal system in man. Neural Plast, 10:93-106

Flowers KA (1976). Visual "closed-loop" and "open-loop" characteristics of voluntary movement in patients with Parkinsonism and intention tremor. Brain, 99(2):269-310

Forman D, Raj A, Buton DC, Power K (2014). Corticospinal excitability of the biceps brachii is higher during arm cycling than an intensity-matched tonic contraction. $\mathrm{J}$ Neurophysiol, 112: 1142-1151

Gantchev N, Viallet F, Aurenty R, Massion J (1996). Impairment of posture-kinetic coordination during initiation of forward oriented stepping movements in parkinsonian patients. Electroencephalography and Clinical Neurophysiology/Electromyography and Motor Control, 101(2):110-120

Geertsen SS, Zuur AT, Nielsen JB (2010). Voluntary activation of ankle muscles is accompanied by subcortical facilitation of their antagonists. J Physiol, 588:2391-2402

Goto Y, Jono Y, Hatanaka R, Nomura Y, Tani Y, Chujo Y, Hiraoka K (2014). Different corticospinal control between discrete and rhythmic movement of the ankle. Frontiers in Human Neuroscience 
Grillner S (1981). Control of locomotion in bipeds, tetrapods, and fish. In: Handbook of Physiology. The Nervous Sysem. Motor Control. Bethesda, D: AM Physiol Soc, sect. 1, vol. 2: 1179-1236

Grillner S, Georgopoulous AP, Jordan LM (1997). Selection and initiation of motor behavior. In: Stein PSG, Grillner S, Selverston AL, Stuart DG (Eds.), Neurones, Networks, and Motor Behavior. MIT Press, Cambridge, Ma, 3-19

Hiraoka K, Abe K. (2007) Cortical and spinal control of ankle joint muscles before and during gait initiation. Somatosensory and Motor Research, 24: 127-133

Hiraoka K, Matuo Y, Iwata A, Onishi T, Abe K. (2006) The effects of external cues on ankle control during gait initiation in Parkinson's disease. Parkinsonism Relat Disord, 12: 97-102

Jane JA, Yashon D, DeMyer W, Bucy PC (1967). The contribution of the precentral gyrus to the pyramidal tract of man. J Neurosurg. 26:244-248

Johnson EO, Vekris MD, Zoubos AB, Soucacos PN (2006). Neuroanatomy of the brachial plexus: the missing link in the continuity between the central and peripheral nervous system. Microsurgery, 26(4): 218-229

Jordan LM (1991). Brainstem and spinal cord mechanisms for the initiation of locomotion. In: Shimamura M, Grillner S, Edgerton VR (Eds.), Neurobiological Basis of Human Locomotion. Japan Scientific Society Press, 3-20

Jordan LM (1998). Initiation of locomotion in mammals. Ann NY Acad Sci, 860: 83-93

Kaneko K, Kawai S, Taguchi T, Fuchigami Y, Morita H, Ofuji A, Yonemura H (1997). Spatial distribution of corticospinal potentials following transcranial electric and magnetic stimulation in human spinal cord. J Neuro Sci, 151: 217-221

Krawitz S, Fedirchuk B, Dai Y, Jordan LM, McCrea DA (2001). State-dependent hyperpolarization of voltage threshold enhances motoneurones excitability during fictive locomotion in the cat. J Physiol, 532:271-281

Lemon RN (2008). Descending pathways in motor control. Annu Rev Neurosci, 31:195218 
Maccabee PJ, Amassian VE, Eberle LP, Cracco RQ. (1993) Magnetic coil stimulation of straight and bent amphibian and mammalian peripheral nerve in vitro: locus of excitation. J Physiol, 460: 201-219

McNeil CJ, Butler JE, Taylor JL, Gandevia SC (2013). Testing the excitability of human motoneurons. Frontiers in Human Neuroscience, 7

Mills KR, Murray NM. (1986) Electrical stimulation over the human vertebral column: which neural elements are excited? Electroencephalogr Clin Neurophysiol, 63: 582-589

Nathan PW, Smith MC (1955). Long descending tracts in man. I. Review of present knowledge. Brain, 78:248-303

Nathan PW, Smith MC, Deacon P (1990). The corticospinal tracts in man. Course and location of fibres at different segmental levels. Brain, 113:303-324

Nikolova M, Pondev N, Christova L, Wolf W, Kossev AR. (2006) Motor cortex excitability changes preceding voluntary muscle activity in simple reaction time task. Eur J Appl Physiol, 98: 212-219

Okada Y, Fukumoto T, Takatori K, Nagino K, Hiraoka K (2011). Abnormalities of the First Three Steps of Gait Initiation in Patients with Parkinson's Disease with Freezing of Gait. Parkinson's Disease, 2011

Perrault MC, Enriquez-Denton M, Hultborn H (1999). Proprioceptive control of extensor activity during fictive scratching and weight support compared to fictive locomotion. J Neurosci, 19:10966-10976

Petersen NT, Taylor JL, Gandevia SC (2002). The effect of electrical stimulation of the corticospinal tract on motor units of human biceps brachii. J Physiol, 544: 277-284

Power KE, Copithorne DB. (2013) Increased corticospinal excitability prior to arm cycling is due to enhanced supraspinal but not spinal motoneurone excitability. Appl. Physiol. Nutr. Metab., 38: 1154-1161

Power KE, McCrea DA, Fedirchuk B (2010). Intraspinally mediated state-dependent enhancement of motoneurone excitability during fictive scratch in the adult decerebrate cat. J Physiol, 15:2839-2857

Pyndt HS, Laursen M, Nielsen JB (2003). Changes in Reciprocal Inhibition Across the Ankle Joint With Changes in External Load and Pedaling Rate During Bicycling. J Neurophysiol, 90:3168-3177 
Pyndt HS, Nielsen JB (2003). Modulation of transmission in the corticospinal and group Ia afferent pathways to soleus motoneurones during bicycling. J Neurophysiol, 89: 304314

Rosin R, Topka H, Dichgans J (1997). Gait initiation in Parkinson's disease. Movement disorders, 12(5): 682-690

Rossignol S (1996). Neural control of stereotypical limb movements. In: Rothwell L, Shepard J (Eds.), Integration of Motor, Circulatory, Respiratory and Metabolic Control During Exercise. American Physiological Society, 173-216

Rossini PM, Di Stefano E, Stanzione P. (1985) Nerve impulse propagation along central and peripheral fast conducting motor and sensory pathways in man. Electroencephalogr Clin Neurophysiol, 60: 320-334

Schmidt BJ (1994). Afterhyperpolarization modulation in lumbar motoneurones during locomotor-like rhythmic activity in the neonatal rat spinal cord in vitro. Exp Brain Res, 99:214-222

Shik ML, Severin RV, Orlovsky GN (1966). Control of walking and running by means of electrical stimulation of the mid-brain. Biophysics, 11:756-765

Sidhu SK, Hoffmann BW, Cresswell AG, Carroll TJ. (2012) Corticospinal contributions to lower limb muscle activity during cycling in humans. J Neurophysiol, 107: 306-314

Skinner RD, Kinjo N, Henderson V, Garcia-Rill E (1990). Locomotor projection from the pedunculopontine nucleus to the spinal cord. Neuroreport, 1:183-186

Taylor JL. (2006). Stimulation at the cervicomedullary junction in human subjects. $J$ Electromyogr Kines, 16: 215-223

Taylor JL, Petersen NT, Butler JE, Gandevia SC. (2002). Interaction of transcranial magnetic stimulation and transmastoid stimulation in human subjects. J Physiol, 541: 949-958

Ugawa Y, Genba-Shimizu K, Kanazawa I. (1995) Electrical stimulation of the human descending motor tracts at several levels, Can J Neurol Sci, 22: 36-42

Ugawa Y, Rothwell JC, Day BL, Thompson PD, Marsden CD (1991). Percutaneous electrical stimulation of corticospinal pathways at the level of the pyramidal decussation in humans. Ann Neurol, 29: 418-427 
Whelan P (1996). Control of locomotion in the decerebrate cat. Prog Neurobiol, 49:481515

Zehr EP, Carroll TJ, Chua R, Collins DF, Frigon A, Haridas C, Hundza SR, Thompson AK (2004). Possible contributions of CPG activity to the control of rhythmic human arm movement. Can J Physiol Pharmacol, 82:556-568

Zehr EP, Hundza SR, Vasudevan EV (2009). The quadrupedal nature of human bipedal locomotion. Exercise and sport sciences reviews, 37: 102-108

Zehr EP, Stein RB (1999). What function do reflexes serve during human locomotion? Progress in Neurobiology, 58: 185-205 


\title{
Chapter 3 Differences in Corticospinal Excitability to the Biceps Brachii Between Arm Cycling and Tonic Contraction Are Not Evident at the Immediate Onset of Movement
}

\author{
Davis Forman ${ }^{1}$, Devin T.G. Philpott ${ }^{1}$, Duane C. Button ${ }^{1,2}$ and Kevin E. Power ${ }^{1,2^{*}}$ \\ ${ }^{1}$ School of Human Kinetics and Recreation, Memorial University of Newfoundland, St. \\ John's, Newfoundland, Canada; \\ ${ }^{2}$ Faculty of Medicine, Memorial University of Newfoundland, St. John's, Newfoundland, \\ Canada
}

Davis Forman, BHSc

Memorial University of Newfoundland

230 Elizabeth Ave, St. John's, NL, A1B 3X9

Devin Philpott, BSc

Memorial University of Newfoundland

230 Elizabeth Ave, St. John's, NL, A1B 3X9

Duane Button, $\mathrm{PhD}$

Memorial University of Newfoundland

230 Elizabeth Ave, St. John's, NL, A1B 3X9

*Corresponding Author

Kevin Power, $\mathrm{PhD}$

Assistant Professor

Kinesiology | School of Human Kinetics and Recreation

Memorial University of Newfoundland

230 Elizabeth Ave, St. John's, NL, A1B 3 X9

Email: kevin.power@mun.ca

Running Head: Corticospinal excitability at the onset of arm cycling

Keywords: initiation, transmastoid, transcranial, MEP, CMEP, steady-state 


\subsection{ABSTRACT}

This is the first study to examine changes in corticospinal excitability to the biceps brachii during the onset of arm cycling from a resting position and at points when steadystate arm cycling had been reached. We hypothesized that corticospinal excitability during the initiation of arm cycling would not be different from a tonic contraction, but that supraspinal excitability would be elevated once arm cycling reached steady-state. Corticospinal and spinal excitability were assessed using motor evoked potentials (MEPs) elicited via transcranial magnetic stimulation (TMS) and cervicomedullary-evoked potentials (CMEPs) elicited via transmastoid electrical stimulation (TMES), respectively. Evoked responses were recorded from the biceps brachii during elbow flexion (6 o'clock relative to a clock face) for both arm cycling and an intensity-matched tonic contraction at three separate periods: 1) immediately at the onset of movement and after the completion of the 2) $4^{\text {th }}$ revolution and 3) $9^{\text {th }}$ revolution. As hypothesized, there was no difference during initiation between tasks for MEP $(P=0.79)$ or CMEP amplitudes $(P=0.57)$. However, MEP amplitudes were significantly larger during arm cycling than an intensitymatched tonic contraction after the completion of the $4^{\text {th }}$ (Cycling: $76.48 \pm 17.35 \%$ of $\mathrm{M}_{\max }$, Tonic: $63.45 \pm 18.45 \%$ of $\mathrm{M}_{\max }, P<0.05$ ) and $9^{\text {th }}$ revolution (Cycling: $72.37 \pm$ $15.96 \%$ of $\mathrm{M}_{\max }$, Tonic: $58.1 \pm 24.23 \%$ of $\left.\mathrm{M}_{\max }, P<0.05\right)$. There were no differences between conditions in CMEP amplitudes $\left(4^{\text {th }}: P=0.31,9^{\text {th }}: P=0.29\right)$. These results demonstrate that corticospinal excitability is not different between the initiation of arm cycling and a tonic contraction, but supraspinal excitability is enhanced during steadystate arm cycling. This suggests that the initial movement of a rhythmic motor output may be under different control than the subsequent, steady-state output. 


\subsection{INTRODUCTION}

Animal research has demonstrated that although afferent and descending input contribute to the production of natural, smooth movement, the basic characteristics for rhythmic motor outputs can be generated solely by spinal circuitry. The essential pattern of alternating activation of functional antagonists has been shown to originate in spinally mediated networks of cells known as central pattern generators (CPGs) (Grillner, 1981; Jordan, 1998). Indirect evidence suggests that rhythmic motor outputs in humans, such as walking, running, and swimming, are also driven by spinally mediated CPGs (Capaday et al. 1999; Zehr \& Stein, 1999; Pyndt and Nielsen, 2003; Carroll et al. 2006; Zehr et al. 2009). However, unlike our quadruped counterparts, supraspinal input is required during CPG-mediated motor outputs (Petersen et al. 2001; Sidhu et al. 2012; Forman et al. 2014).

A common method of deducing the underlying mechanisms that are responsible for generating rhythmic movements in humans has been to compare measures during rhythmic tasks with those taken during a tonic contraction. These two motor outputs are compared because the generation of the basic rhythmic and alternating pattern of muscle activity during arm cycling is generated, in part, by spinal interneuronal networks (Zehr et al., 2004). A tonic contraction of similar muscle groups is chosen to represent a similar level of motoneurone pool activation, but with reduced or absent activation of spinal interneuronal groups contributing to the generation of rhythmic activation during arm cycling. For example, we recently assessed corticospinal and spinal excitability of the biceps brachii during arm cycling and an intensity-matched tonic contraction (Forman et al. 2014). In this study, measurements were taken after participants had reached, and were 
maintaining, a constant cycling cadence of 60 RPM. However, it is unlikely that the corticospinal modulation observed during steady-state cycling can be generalised to other components of the movement, such as initiation, cessation or acceleration of arm cycling. Some of our previous work supports this statement. We have previously demonstrated that supraspinal, not spinal excitability to the biceps brachii is enhanced prior to arm cycling, but this enhanced excitability is not different from an intensity-matched tonic contraction (Copithorne et al. 2014). This differs from our findings during arm cycling, which demonstrated that corticospinal and spinal excitability are both higher during arm cycling than a tonic contraction (Forman et al. 2014). These findings indicate that a transition in corticospinal excitability from pre-movement to movement must occur at some point once the movement begins.

Research suggests that this transition may not happen immediately at movement onset. Hiraoka and Abe (2007) demonstrated that the corticospinal modulation of the soleus and the tibialis anterior (TA) observed prior to the initiation of gait persists once gait is initiated. Additionally, differences observed in corticospinal excitability to the muscles of the ankle between discrete and rhythmic ankle movement present in the later stages of rhythmic ankle movement, but not in the beginning (Goto et al. 2014). Providing indirect support for this idea are results from Parkinson's disease studies that have found abnormalities in the first step of gait initiation but not in subsequent steps (Okada et al. 2011). These findings indicate that the initiation and steady-state phases of rhythmic motor outputs may be under different neural control. To the best of our knowledge, human studies have not characterized the progression of corticospinal excitability from the initiation of arm cycling to steady-state. 
The purpose of the present study was to examine corticospinal excitability of the biceps brachii from the onset of arm cycling to a point where a constant cadence was reached in comparison to an intensity-matched tonic contraction. Corticospinal and spinal excitability were assessed in the biceps brachii using TMS-evoked MEPs and TMESevoked CMEPs, respectively. Measures were taken at three points throughout the movement: 1) during the initiation of arm cycling, 2) after the completion of the 4th revolution, and 3) after the completion of the 9th revolution (at which time, participants had reached a constant cycling cadence; steady-state). We hypothesized that there would be no differences in either corticospinal or spinal excitability during the initiation of arm cycling, but that supraspinal excitability would be enhanced during the $4^{\text {th }}$ and $9^{\text {th }}$ revolutions. 


\subsection{METHODS}

\subsubsection{Ethical Approval}

Procedures were verbally explained to the volunteers and written consent was obtained prior to participation in the study. This study was conducted in accordance with the Helsinki declaration and approved by the Interdisciplinary Committee on Ethics in Human Research at Memorial University of Newfoundland (ICEHR\# 20150990-HK). Procedures were in accordance with the Tri-Council guideline in Canada and potential risks were fully disclosed to participants.

\subsubsection{Participants}

Ten, male volunteers $(22.8 \pm 1.8$ years of age, $85.3 \pm 7.3 \mathrm{~kg}, 9$ right handed and 1 left handed) were recruited for this study. Participants were excluded from participation if they had any known neurological impairments or were unfit for vigorous physical activity. Prior to the experiment, all volunteers were required to complete a magnetic stimulation safety-checklist in order to screen for contraindications to magnetic stimulation (Rossi et al. 2009). Additionally, participants were asked to complete a Physical Activity Readiness Questionnaire for Everyone (PAR-Q+; Canadian Society for Exercise Physiology (CSEP)) to screen for any contraindications to exercise or physical activity.

\subsubsection{Experimental Set-up}

This study was carried out with the use of an arm cycle ergometer (Monark Rehab Trainer, model 881E). Participants were seated upright and placed at a specific, but 
comfortable distance from the hand pedals so that the elbows did not fully extend during cycling. This ensured that there was no variation in trunk posture during movement (leaning forward or back). Seat height was adjusted so that the shoulders of each individual were approximately the same height as the crank shaft of the ergometer. The hand pedals of the ergometer were fixed 180 degrees out of phase for the entire duration of the protocol. Prior to all cycling trials, participants were given braces for their wrists in order to limit wrist flexion/extension during cycling. This was done to better isolate the biceps brachii as heteronymous reflex connections exist between the wrist flexors and biceps brachii (Manning and Bawa, 2011).

For this study, measurements were taken at a single crank position; 6 o'clock relative to a clock face, whereby 6 o'clock was defined as the "bottom dead centre" of a full revolution. This position was made relative to the hand dominance for each individual. For example, 6 o'clock for a right handed participant occurred when their right hand was positioned at "bottom dead centre" of the arm crank. For a left handed individual, 6 o'clock occurred when their left hand was positioned at "bottom dead centre." This position represents the most active phase of the biceps brachii during arm cycling as it occurs during the portion of arm cycling where the elbow is flexing.

Measurements were taken during two separate motor tasks; arm cycling and an intensity-matched tonic contraction. In this context, 'intensity-matched' refers to the fact that the EMG of the biceps brachii was the same for each motor task. For arm cycling, participants started at rest with their dominant hand at the 5 o'clock position (the position that immediately precedes peak biceps brachii activity). Following the initiation of arm cycling, measurements were then taken from three separate periods at the 6 o'clock 
position; 1) immediately after the initiation of pedal movement, as the pedal travelled from 5 o'clock to 6 o'clock (defined as movement initiation), 2) after 4 complete revolutions had occurred (at which point the participants were accelerating), and 3) after 9 complete revolutions had occurred. Upon completing 9 revolutions, participants had reached a consistent cycling cadence of 60 RPM, and this measurement was defined as 'steady state.' For the sake of simplicity, these points throughout the movement will henceforth be referred to as the following: measurements taken during initiation (as the pedal first travels from 5 to 6 o'clock) will be labeled as 'condition 1'; measurements taken during the completion of the $4^{\text {th }}$ revolution will be labeled as 'condition 2 '; and measurements taken during the completion of the $9^{\text {th }}$ revolution will labeled as 'condition 3'. The order of these conditions was randomized and responses were elicited automatically as the hand pedal passed the 6 o'clock position. Following arm cycling, the ergometer pedals were locked in place and measurements were taken while participants produced a tonic contraction that was matched to the EMG of each of the three respective cycling conditions.

\subsubsection{Electromyography Recording}

EMG activity of the biceps brachii, triceps brachii, extensor carpi radialis (ECR) and the flexor carpi radialis (FCR) of the dominant arm was recorded using pairs of surface electrodes (MeditraceTM 130 ECG conductive adhesive electrodes). Electrodes were positioned over the midline of the biceps brachii, on the lateral head of the triceps brachii, and over the midline of the FCR and ECR. A ground electrode was placed on the lateral epicondyle of the tested limb. Prior to electrode placement over recording sites, the 
skin was thoroughly prepared by removal of dead epithelial cells (using abrasive paper) followed by sanitization with an isopropyl alcohol swab. EMG was collected on-line at 5 KHz using CED 1401 interface and Signal 4 (Cambridge Electronic Design Ltd., Cambridge, UK) software program. Signals were amplified (gain of 300) and filtered using a 3-pole Butterworth with cut-off frequencies of 10-1000 Hz.

\subsubsection{Stimulation Conditions}

Motor responses were recorded from the biceps brachii using three separate stimulation techniques: brachial plexus electrical stimulation at Erb's point; TMES; and TMS. All volunteers had prior experience with all three stimulation procedures. The intensities of the stimulations were determined while participants had their hands placed on the hand pedals of the ergometer. Their dominant hand was placed at the 6 o'clock position and their non-dominant hand at the 12 o'clock position. In this posture, participants were then instructed to produce $5 \%$ of their biceps brachii maximal voluntary EMG (MVE; described below) by pulling against the locked handles. It was chosen to establish stimulation intensities during an active contraction instead of at rest as the experimental conditions were active motor outputs. Matched potentials determined at rest may become unmatched once they are evoked in a voluntary task.

In order to determine each participant's MVE, the activation level of all four muscles were assessed during a maximal, 10 second, arm cycling sprint. Participants were seated behind a table-mounted, Monark Wingate Testing Ergometer (model 849E) that had been fitted with hand pedals. After a 2 minute warm-up of arm cycling at a selfselected pace, participants were instructed to begin cycling as fast and as hard as they 
could. Once they reached a cadence of 100 RPM, a load of 5\% of the individual's bodyweight was automatically applied to the pedals. Participants then continued cycling at maximal effort for 10 seconds. From this trial, peak power (PP) and MVE of the four muscles were determined. MVE was calculated by taking a root mean square (RMS) of the raw EMG using a $25 \mathrm{~ms}$ moving average. Unlike isometric maximal voluntary contractions (MVCs) where EMG is relatively consistent, maximal cycling results in phases of high muscle activity and low muscle activity, with a peak occurring once every revolution. The maximum value of each of these peaks from the RMS signal was summed and averaged from the middle 4 seconds of the 10 second trial (3-7 seconds; approximately 8-10 peaks depending on the individual). The middle 4 seconds were chosen to avoid measurements during the initial phase involved in overcoming the added resistance and to avoid potential fatigue effects towards the end of the 10 second sprint. Following 10 minutes of rest, participants were then moved over to the Monark Rehab Trainer to determine the stimulation intensities that were used for the rest of the experiment. For all stimulations, participants were instructed to produce $5 \%$ of their biceps brachii MVE by pulling against the locked pedals (i.e. tonic elbow flexion). Participants were shown a horizontal line on a computer monitor equal to the $5 \%$ biceps brachii MVE and were asked to reach and maintain that line. Stimulations were elicited during this activity (see below).

\subsubsection{Brachial Plexus Stimulation}

The $\mathrm{M}_{\max }$ of the biceps brachii was first determined by eliciting M-waves through electrical stimulation of the brachial plexus at Erb's point (DS7AH, Digitimer Ltd., 
Welwyn Garden City, Hertfordshire, UK). A pulse duration of $200 \mu$ s was used and intensities ranged from $80-250 \mathrm{~mA}$. The cathode was placed in the supraclavicular fossa and the anode on the acromion process. The initial stimulation intensity was set at $25 \mathrm{~mA}$ and gradually increased until the elicited M-waves of the biceps brachii reached a plateau. The plateau stimulation was then increased by $10 \%$ to ensure maximal M-waves were elicited throughout the study.

\subsubsection{TMES}

TMES was delivered using $\mathrm{Ag}-\mathrm{AgCl}$ surface electrodes applied just inferior to the mastoid processes. The pulse duration was set at $100 \mu$ s and stimulation intensities ranged between 150-275mA (DS7AH, Digitimer Ltd., Welwyn Garden City, Hertfordshire, UK). Stimulation intensity started at $25 \mathrm{~mA}$ and was gradually increased until the average of 8 CMEP amplitudes fell within the target limit. For this study, the acceptable target range was $15-20 \%$ of the individual's $M_{\max }$ (determined above). This stimulation intensity was then used throughout the remainder of the experiment.

\subsubsection{TMS}

MEPs were elicited via TMS using a Magstim 200 (Magstim, Dyfed, UK). Stimulations were delivered over the vertex with a circular coil $(13.5 \mathrm{~cm}$ outside diameter). Vertex was determined by measuring the mid-point between the participant's nasion and inion, and the mid-point between the participant's tragi. The intersection of these two points was measured, marked and defined as vertex (Power and Copithorne, 2013). The coil was held tangentially to the participant's skull, or approximately parallel 
to the floor, with the direction of the current flow preferentially activating either the left or right motor cortex (depending on hand dominance; left for right handed individual's and right for left handed individual's). The coil was held firmly against the participant's head by one of the investigators to ensure careful and consistent alignment over vertex for each trial. Stimulation intensity started at approximately $25 \%$ of maximum stimulator output (\%MSO) and was gradually increased until the average of 8 MEP amplitudes matched the average CMEP amplitude previously determined (i.e. approximately 15-20\% of the individual's $\mathrm{M}_{\max }$ ). This \% MSO was then used throughout the remainder of the experiment.

\subsubsection{Experimental Protocol}

After the stimulation intensities were determined, the hand pedals were unlocked and a resistance of 5\% of the individual's PP (determined from the 10 -second sprint) was set on the ergometer. Participants were positioned with their dominant hand at the 5 o'clock position (immediately before 6 o'clock where they would be receiving stimulation) and their non-dominant hand at 11 o'clock. They were then instructed to begin cycling based on external cues from an automated audio program (Audacity 2.0.5). A preparation tone was first given (frequency of $200 \mathrm{~Hz}$; duration, $100 \mathrm{~ms}$ ), followed one second later by a 'go' tone (frequency of $100 \mathrm{~Hz}$; duration, $100 \mathrm{~ms}$ ). Upon hearing the 'go tone,' participants were told to begin cycling at a comfortable pace and were asked to reach and maintain a target cadence of 60 RPM. A final tone (frequency of $300 \mathrm{~Hz}$; duration, 100ms) was then given 15 seconds after the 'go' tone, which was a signal to the 
participant to cease cycling. This time period was considered a single trial with one stimulation elicited per trial.

Two M-waves were first elicited during arm cycling for each of the three conditions (condition 1, condition 2, and condition 3; 6 stimulations total). The conditions were randomized and participants were not told when they would be receiving the stimulation. Following this, 6 MEPs and 6 CMEPs were then delivered during arm cycling for each condition, with an additional 10 trials void of stimulation (46 trials total). Their order was randomized and participants were not informed which stimulation (if any) they would be receiving. Finally, 2 additional M-waves were delivered randomly during arm cycling at each condition.

Pre-stimulus EMG of the biceps brachii (defined as a $50 \mathrm{~ms}$, rectified average of the EMG activity immediately prior to the point of stimulation) was measured following all arm cycling trials (Forman et al. 2014). These values were then grouped and averaged according to the condition in which they occurred (1-3). The purpose of this step was to match the EMG between motor tasks as muscle activity can influence evoked responses. Once found, the hand pedals were once again locked with the dominant hand placed at the 6 o'clock position. Participants were shown a horizontal line in an RMS channel on a computer monitor that reflected the pre-stimulus EMG value for a given condition. Participants were instructed to match that line as closely as possible by pulling against the locked handles. A total of 10 MEPs and 10 CMEPs were delivered randomly within the same configuration, followed by a separate configuration of 3 M-waves. Together, this represented a single tonic condition, which coincided with one cycling condition (3 in total). The order of the tonic conditions was randomized. 


\subsubsection{Measurements}

Data was analyzed off-line using Signal 4 software (CED, UK). The peak-to-peak amplitudes of MEPs, CMEPs and M-waves of the biceps brachii were measured. The peak-to-peak amplitudes for all evoked potentials were measured from the initial deflection of the voltage trace from the background EMG to the return of the trace to background levels. Because changes in MEP and CMEP amplitudes can be the result of changes to the M-wave, both MEPs and CMEPs were normalized to the M-wave evoked during the same experimental condition. Corticospinal silent periods (CSPs) were also assessed, and were measured from the start of the stimulation artifact to the return of voluntary muscle activity following the evoked MEP (visually determined). Measurements were taken from individual files and averaged afterwards.

\subsubsection{Statistics}

Statistics were performed using IBM's SPSS Statistics Version 19. A paired t-test was used to examine differences in the initial, matched MEP and CMEP amplitudes (normalized to M-wave). One-way (condition) repeated-measures ANOVAs assessed whether statistically significant differences in MEP and CMEP amplitudes (normalized to M-wave), CSPs and pre-stimulation values occurred during arm cycling or tonic contractions across conditions. Separate, paired t-tests were then used to examine differences in measures between arm cycling and intensity matched tonic contractions. All statistics were run on group data and a significance level of $P<.05$ was used. All data is reported as means $\pm \mathrm{SD}$ and illustrated in figures as SE. 


\subsection{RESULTS}

\subsubsection{EMG Patterns During Arm Cycling}

The rectified EMG of a single participant for the biceps brachii and triceps brachii during arm cycling can be seen in Fig. 1A. In this example, eight frames without stimulation were rectified and averaged over a 1 second window, with the participant cycling at a constant cadence of 60 RPM. At this cadence, 1 second represents one full revolution of arm cycling. The black arrow in the figure demonstrates the point where stimulations were delivered; when the elbow is flexing and the biceps brachii is most active.

\subsubsection{Matched Stimulations}

Fig. 1B shows group MEP and CMEP amplitudes taken during the initial setup where participants produced a tonic contraction of 5\% of their maximum biceps brachii EMG; determined from the 10-second, maximal cycling sprint (see Methods). The target range for each participant was $15-20 \%$ of the individual's $\mathrm{M}_{\max }$. There was not a significant difference between MEP and CMEP amplitudes $(P=0.579)$.

\subsubsection{Corticospinal Excitability}

Fig. 2 shows a representative example of the MEP amplitude differences between arm cycling and an intensity-matched tonic contraction for the three different conditions. For this participant, the average MEP amplitude elicited during condition 1 for arm cycling was $70.44 \%$ of $\mathrm{M}_{\max }$ compared to $70.57 \%$ of $\mathrm{M}_{\max }$ during an intensity-matched tonic contraction. MEP amplitude during condition 2 for arm cycling was $71.38 \%$ of 
$\mathrm{M}_{\max }$, compared to $64.36 \%$ of $\mathrm{M}_{\max }$ for the intensity-matched tonic contraction, and finally, MEP amplitude during condition 3 for arm cycling was $76.95 \%$ of $\mathrm{M}_{\max }$ and $35.41 \%$ for the intensity-matched tonic contraction. Group data (Fig. 3A) revealed that there were no differences in MEP amplitudes between arm cycling and an intensitymatched tonic contraction for condition $1(P=0.79)$. MEP amplitudes elicited during condition 2 for arm cycling were significantly larger compared to an intensity-matched tonic contraction (Cycling: $76.48 \pm 17.35 \%$ of $\mathrm{M}_{\max }$, Tonic: $63.45 \pm 18.45 \%$ of $\mathrm{M}_{\max }, P<$ 0.05). MEP amplitudes were also significantly larger during condition 3 for arm cycling compared to an intensity-matched tonic contraction (Cycling: $72.37 \pm 15.96 \%$ of $\mathrm{M}_{\max }$, Tonic: $58.1 \pm 24.23 \%$ of $\left.\mathrm{M}_{\max }, P<0.05\right)$. A one-way, repeated measures ANOVA showed that there was no main effect of condition within arm cycling $(P=0.67)$. There was also no main effect of condition within the intensity-matched tonic contraction $(P=$ $0.067)$.

Contraction intensity has a large influence on the evoked responses from both TMS and TMES. It is therefore vitally important that the pre-stimulus EMG between cycling and tonic tasks are not different. Figures $3 \mathrm{~B}$ and $\mathrm{C}$ show the results of prestimulus EMG produced prior to TMS for the biceps and triceps brachii, respectively. There were no differences in the biceps brachii pre-stimulus EMG between the cycling and tonic tasks for any condition (condition 1: $P=0.676$; condition $2: P=0.862$; condition 3: $P=0.738$ ). However, there was a main effect of condition, with the biceps brachii pre-stimulus EMG decreasing throughout the movement during arm cycling (condition 1: $16.36 \pm 5.02 \%$ of maximum, condition 2: $13.0 \pm 3.03 \%$ of maximum, condition $3: 11.18 \pm 3.89 \%$ of maximum, $\mathrm{P}<0.05)$ and for the intensity matched tonic 
contractions (condition 1: $15.98 \pm 4.31 \%$ of maximum, condition 2: $13.09 \pm 3.25 \%$ of maximum, condition 3: $11.0 \pm 3.44 \%$ of maximum, $P<0.05$ ). There was no difference in pre-stimulus EMG of the triceps brachii between tasks for condition 1 or condition 3 (condition 1: $P=0.142$; condition 3: $P=0.163$ ). There was, however, a significant difference in triceps brachii pre-stimulus EMG for condition 2, with EMG during the intensity-matched tonic contraction larger than arm cycling (Cycling: $4.99 \pm 1.2 \%$ of maximum, Tonic: $6.68 \pm 2.55 \%$ of maximum, $P<0.05$ ). There was no main effect of condition for triceps brachii pre-stimulus EMG for arm cycling $(P=0.071)$ or for the intensity-matched tonic contractions $(P=0.072)$. However, while the differences across conditions for triceps brachii pre-stimulus EMG were not significant, the EMG followed a similar pattern as the biceps brachii pre-stimulus EMG (decreasing across conditions; Figures 3B and C).

\subsubsection{Spinal Excitability}

In order to differentiate supraspinal and spinal influences on changes to MEP amplitudes between tasks, we examined CMEPs as a measure of spinal excitability. Fig. 4A shows the group data for CMEPs elicited during both tasks for the three conditions. There were no differences between arm cycling and the intensity-matched tonic contraction at for condition $1(P=0.57)$, condition $2(P=0.311)$, or condition $3(P=$ 0.291). There was also no main effect of condition for arm cycling $(P=0.926)$ or intensity-matched tonic contraction $(P=0.09)$.

Figures 4B and $\mathrm{C}$ show the results of pre-stimulus EMG produced during TMES

trials for the biceps and triceps brachii, respectively. As with MEPs, contraction intensity 
can influence the evoked CMEP amplitude following TMES. Thus, in order to compare CMEPs between tasks, the pre-stimulus EMG must be matched. There were no differences in pre-stimulus EMG in the biceps brachii between arm cycling and the intensity-matched tonic contraction during condition $1(P=0.979)$, condition $2(P=$ $0.539)$ or condition $3(P=0.615)$. There were also no main effects of condition for arm cycling $(P=0.223)$ or intensity-matched tonic contractions $(P=0.066)$. There were no differences in triceps brachii pre-stimulus EMG between tasks for condition $1(P=$ 0.304), condition $2(P=0.415)$, or condition $3(P=0.154)$. There was also no main effect of condition for either arm cycling $(P=0.232)$ or the intensity-matched tonic contractions $(P=0.185)$

\subsubsection{CSPS}

CSPs were measured as the duration of time between the stimulation artifact, produced via TMS, to the reappearance of voluntary EMG in the biceps brachii. Fig. 5 depicts the results of group data for CSPs measured for both tasks during the three conditions. There was no difference in CSP duration between tasks for condition $1(P=$ 0.891). The CSP duration was significantly longer during arm cycling than the intensitymatched tonic contraction for condition 2 (Cycling: $83.91 \pm 12.76 \mathrm{~ms}$, Tonic: $74.34 \pm$ 11.69ms, $P<0.05$ ) and condition 3 (Cycling: $84.46 \pm 12.84 \mathrm{~ms}$, Tonic: $73.08 \pm 14.24 \mathrm{~m}, P$ $<0.05)$. There was also a main effect of condition for arm cycling, with CSP duration increasing throughout the movement (condition 1: $71.04 \pm 12.03 \mathrm{~ms}$, condition 2: $83.91 \pm$ $12.76 \mathrm{~ms}$, condition 3: $84.46 \pm 12.84 \mathrm{~ms}, P<0.05)$. There was no effect of condition on the intensity-matched tonic contractions $(P=0.322)$. 


\subsection{DISCUSSION}

This study is the first to demonstrate that corticospinal excitability of the biceps brachii is similarly modulated at the onset of arm cycling and an intensity-matched tonic contraction. This was established by a lack of change between tasks in MEP amplitude (Fig. 3A), CMEP amplitude (Fig. 4A) and CSP duration (Fig. 5A). However, these similarities between motor outputs did not persist indefinitely as we found that corticospinal excitability was modulated differently between arm cycling and an intensity-matched tonic contraction during conditions 2 and 3 . This was demonstrated via larger MEP amplitudes (Fig. 3A) and longer CSP durations (Fig. 5A) during arm cycling trials. Spinal excitability did not follow the same trend, as CMEP amplitudes (Fig. 4A) were not significantly different during condition 2 and 3 between tasks, indicating that the changes in corticospinal excitability were supraspinally mediated. These results suggest that similar to premovement changes in corticospinal excitability, there appears to be a common neural drive used to initiate both arm cycling and tonic contraction. Conversely, once arm cycling has begun (i.e. inertia overcome and accelerating to a predetermined cadence) supraspinal excitability increases to a larger degree than during an intensitymatched tonic contraction, suggesting differences in the neural control of both motor outputs.

\subsubsection{Cycling Initiation}

Supraspinal and spinal excitability of the biceps brachii during the initiation of arm cycling does not appear to be different compared to an intensity-matched tonic contraction (Fig. 3A). The findings of the current study are similar to previous work we 
have done regarding pre-movement changes in corticospinal excitability (Copithorne et al. 2014). In that study, we assessed corticospinal and spinal excitability in the biceps brachii $25 \mathrm{~ms}$ prior to the initiation of both arm cycling and an intensity-matched tonic contraction. We demonstrated that MEP onset latency prior to arm cycling was shorter and MEP amplitude larger relative to resting control. No changes were observed in either CMEP latency or CMEP amplitude, indicating that the enhancement in corticospinal excitability prior to arm cycling was supraspinally mediated. However, there were no differences found between arm cycling and an intensity-matched tonic contraction. These findings suggest that the central processes involved in motor output preparation were similar for the two motor outputs, regardless of how different they may be once they have begun (Forman et al. 2014). This led to our current objective and the purpose of the present study, which was to determine when these differences in supraspinal and spinal excitability arise when transitioning from movement initiation to steady-state motor output.

Our findings indicate that this transition of corticospinal excitability from premovement to during movement does not occur immediately at movement onset as the evoked responses recorded during arm cycling were similar to an intensity-matched tonic contraction at condition 1. This suggests that the patterns of corticospinal modulation observed prior to movement (Copithorne et al. 2014) continue once the motor outputs are initiated. Research on corticospinal contributions to the initiation of gait support this statement. Hiraoka and Abe (2007) assessed supraspinal and spinal excitability to the muscles of the ankle before and during gait initiation. They found that spinal excitability to the soleus was depressed prior to movement, but supraspinal excitability to both the 
soleus and the tibialis anterior (TA) was enhanced. These pre-movement changes in corticospinal excitability were then maintained once gait was initiated. More recently, Hiraoka and colleagues (Goto et al. 2014) examined corticospinal and spinal excitability of the soleus and the TA between two different motor tasks; rhythmic and discrete ankle movement. They demonstrated that there was no difference in corticospinal or spinal excitability between discrete movement and the first cycle of rhythmic movement, indicating that neither supraspinal nor spinal excitability were mediated differently between tasks. However, TA MEPs elicited during the $10^{\text {th }}$ cycle of rhythmic ankle movement were significantly larger than MEPS elicited during discrete ankle movement. The authors surmised that the initial cycle and the forthcoming steady-state cycles must therefore be under different control.

Clinical research of Parkinson's disease (PD) may provide support for this conclusion. A common, clinical feature of PD is bradykinesia, which often manifests as a difficulty for people afflicted with the disease to initiate gait (Gantchev et al. 1996; Rosin et al. 1997). Abnormalities of gait initiation in people with PD have been previously described. Okada et al. (2011) demonstrated that the duration of the initial step in patients with PD is significantly longer compared to healthy controls. Interestingly, no differences in duration were observed in the subsequent steps, indicating that certain motor pathways affected by PD are specific to the initiation of movement but not the rhythmic motor output that follows. These findings suggest that some of the supraspinal pathways affected in people with PD are modulated differently in the healthy population depending on the phase of the forthcoming movement (initiation vs. steady-state). Thus, it could be possible that the initiation of a rhythmic motor output is under the same, supraspinal 
control as a tonic motor output. This may help explain the similarities in corticospinal excitability observed in the present study.

While we propose that the modulation of supraspinal excitability during the initiation of arm cycling is similar to an intensity-matched tonic contraction, it is also worth noting the similarities of the two motor outputs from a spinal perspective. During movement initiation, arm cycling is not truly a rhythmic movement. It does not display the characteristic, alternating activation of functional antagonists, and thus whether or not the spinal CPG is truly engaged at this point of the motor output is questionable (Grillner, 1981; Jordan, 1998). Afferent input may also be similar between the two tasks. Activation of group Ia afferents takes place during arm cycling, which involves the rapid stretching and shortening of the biceps and triceps brachii as the motor output alternates between elbow flexion and extension. While their influence during cycling is mitigated by reciprocal inhibition, they still provide afferent feedback throughout the movement (Tanaka 1974; Crone et al. 1987; Nielsen et al. 1992). However, both the amplitude and the rate of stretch that would have occurred when measurements were taken during the condition 1 for arm cycling would have been diminished in comparison to steady-state arm cycling. In fact, the subtle movement of the upper limbs during the initiation of arm cycling may have been comparable to the minor movement seen during a tonic contraction. If this was the case, then the influence of group Ia afferents between the two tasks may have been similar. The modulation of pre-synaptic inhibition may also follow a comparable pattern between the two motor outputs. Suppressed H-reflex amplitudes have previously been documented during rhythmic arm cycling compared to intensity-matched tonic contractions (Carroll et al. 2006). This is thought to be the result of an increase in 
pre-synaptic inhibition to afferent input due to the activation of spinal CPGs. Considering the non-rhythmic nature of the initiation of arm cycling, pre-synaptic inhibition may follow a pattern of modulation that is similar during a tonic motor output. Additionally, the relative contributions that the muscles of the arm provide to each of the two motor tasks, which are different during steady-state arm cycling compared to a tonic contraction (see Fig. 3C; Fig. 3C and 4C in Forman et al. 2014) are more closely matched between the two tasks at initiation. Indeed, the activity of triceps brachii in the present study, which was not controlled for during collection, was not significantly different between the two outputs for condition 1. This suggests that reciprocal inhibition, which has been shown to be task-dependent in the lower limb (Pyndt et al. 2003) is not likely different between the tasks used in the present study.

\subsubsection{Steady-State Cycling}

In the present study, supraspinal, but not spinal excitability was significantly higher during arm cycling at condition 3 (steady-state) compared to an intensity-matched tonic contraction. This was demonstrated by an increase in the size of the MEP amplitudes (Fig. 3A) but no difference in CMEPs (Fig. 4A). The current results replicate our previous findings (Forman et al. 2014). In that study, TMS and TMES were delivered at three different crank positions (12,3 and 6 o'clock relative to the face of a clock) while participants arm cycled at a constant cadence of 60 RPM and while participant's produced intensity-matched tonic contractions. As was the case in the present study, MEP amplitudes were significantly larger during arm cycling at the 6 o'clock position than during an intensity-matched tonic contraction. No differences in CMEP amplitudes were 
observed between the two motor outputs. However, this was not the first time that corticospinal excitability during steady-state arm cycling had been investigated. Carroll et al. (2006) demonstrated a reduction in corticospinal excitability to the flexor carpi radialis (FCR) during the flexion phase of arm cycling compared to an intensity-matched tonic contraction. This was accompanied by a decrease in the H-reflex. The apparent differences in modulation between the biceps brachii and the FCR may result from the dissimilar function of each muscle. During arm cycling, the purpose of the FCR is mainly to stabilize the wrist, while the biceps brachii is a prime mover that exhibits a strong phase-dependency (see Fig. 1A). It is thus strongly suggested that corticospinal excitability during arm cycling is muscle, or function, dependent. Previous literature involving TMS-induced MEPs in the lower limb supports this statement (Capaday et al. 1999; Sidhu et al. 2012). Further evidence can be found in the comparison of the biceps brachii during arm cycling and the soleus during leg cycling, both of which are considered prime movers in their respective cycling tasks. Pyndt and Nielsen (2003) determined that MEP amplitudes of the soleus were larger during the propulsion phase (downstroke) of leg cycling, which is consistent with our own findings in the biceps brachii. We, along with Pyndt and Nielsen (2003) suggested that this increase in MEP amplitude is due to enhanced excitability of cortical neurones, thus facilitating their activation via TMS. From a motor control standpoint, this may be a mechanism for the motor cortex to ensure adequate muscle activation to the prime mover during the propulsive phase of cycling.

One measure that was not conducted in our earlier work, but was included in the present study is CSPs following TMS. According to Fig. 5, CSP duration was not 
different between tasks for condition 1, but was significantly longer during arm cycling for conditions 2 and 3 . This suggests the possibility that the mediation of $\mathrm{GABA}_{\mathrm{A}}$ inhibition, which is a contributing factor to CSP duration, may be task-dependent (Paulus et al. 2008). However, a more plausible theory comes from the work of Orth and Rothwell (2004) who have previously demonstrated a positive association between the size of MEP amplitudes and the length of CSP duration. Considering the similar patterns of MEP amplitude (Fig. 3A) and CSP duration (Fig. 5) between tasks and across conditions in the present study, whereby larger MEPs appear to be associated with longer CSP durations, this may be the most reasonable explanation. While we do not rule out the possibility that the changes in CSPs may have been due to differences in modulation between tasks, it would be pre-mature to draw such a conclusion based on available evidence. This emphasizes the need for additional work and future research should include independent measures of surpaspinal pathways.

\subsubsection{Acceleration}

Alongside steady-state arm cycling, we also assessed corticospinal excitability during acceleration in the present study. This was done via measurements taken during condition 2. Although participants were instructed to cycle comfortably up to a target cadence of 60 RPM, we were able to visually confirm that they had not yet reached that target by the $4^{\text {th }}$ revolution. Thus, participants were accelerating from a resting position during condition 2. Our reasoning for taking this measurement was that it was mid-way between initiation (condition 1) and steady-state arm cycling (condition 3). We reasoned that it could therefore provide potential insight into how the modulation of corticospinal 
excitability progresses once arm cycling is initiated. Our findings revealed that corticospinal excitability during condition 2 was similar to steady-state cycling (condition 3) with MEP amplitudes larger than an intensity-matched tonic contraction (Fig. 3A) with no difference in CMEP amplitudes (Fig. 4A). As with our findings during condition 3, this indicates that an increase in supraspinal, not spinal excitability likely occurred. Two important conclusions can be drawn from these results. The first is that by the time an individual completes four revolutions, the movement has likely become rhythmic as corticospinal excitability is modulated similarly to steady-state cycling. This indicates that the transition of corticospinal modulation from the initial moments of arm cycling to the modulation of steady-state arm cycling must occur within the first four revolutions. Further research is required to establish a more detailed time-course of this progression. Second, it seems that once arm cycling has become rhythmic, the stability of the motor output from a cadence perspective (either constant or accelerating) does not appear to influence corticospinal excitability when compared to a tonic contraction. This can be seen in the similarities of the evoked responses at condition 2 (acceleration) and condition 3 (steady-state). The case may be that cadence does not influence corticospinal excitability, or that the difference in cadence between the $4^{\text {th }}$ and $9^{\text {th }}$ revolution was too small to significantly modulate the MEP amplitudes.

\subsection{CONCLUSION}

Neither supraspinal nor spinal excitability were different between the initiation of arm cycling and an intensity-matched tonic contraction, whereas an increase in supraspinal excitability was observed in the later phases of the movement $\left(4^{\text {th }}\right.$ and $9^{\text {th }}$ 
revolution). This indicates that there is a commonality in how the CNS initiates motor outputs in humans, regardless of how different those outputs may be. Consequently, it can be suggested that the initial moments of arm cycling are likely under different central control than arm cycling once it has reached steady-state. This change in modulation appears to originate at the supraspinal level and occurs prior to the completion of the $4^{\text {th }}$ revolution. Further research is required to establish a more robust understanding of how this modulation progresses throughout rhythmic motor tasks. 


\subsection{FIGURE LEGEND}

Figure 1. A: Rectified EMG values of a single participant throughout a single revolution of arm cycling. The black arrow signifies the 6 o'clock position. B: Group data (mean \pm $\mathrm{SE}, \mathrm{n}=7$ ) for matched MEP amplitudes (black bar) and CMEP amplitudes (white bar) during initial setup of the experiment. Amplitudes are expressed as a $\%$ of the $\mathrm{M}_{\max }$.

Figure 2. Average traces of 6 MEPs during arm cycling (solid black lines) and intensitymatched tonic contractions (solid grey lines) taken during the three conditions of a single participant.

Figure 3. Group data (mean $\pm \mathrm{SE}, \mathrm{n}=10$ ) for A) MEP amplitude, B) pre-stimulus EMG of the biceps brachii, and C) pre-stimulus EMG of the triceps brachii. Values from arm cycling (black bars) and intensity-matched tonic contractions (white bars) are shown for the three conditions. MEP amplitudes are expressed relative to the $\mathrm{M}_{\max }$ taken during the same condition and EMG is expressed relative to the maximum EMG found during the 10 second, maximal arm cycling sprint. Asterisks denote a significant difference $(\mathrm{P}<0.05)$ between arm cycling and the intensity-matched tonic contraction.

Figure 4. Group data (mean $\pm \mathrm{SE}, \mathrm{n}=7$ ) for A) CMEP amplitude, B) pre-stimulus EMG of the biceps brachii, and C) pre-stimulus EMG of the triceps brachii. Values from arm cycling (black bars) and tonic contractions (white bars) are shown for the three conditions. CMEP amplitudes are expressed relative to the $\mathrm{M}_{\max }$ taken during the same condition, and EMG is expressed relative to the maximum EMG found during the 10 second, maximal arm cycling sprint. Asterisks denote a significant difference $(\mathrm{P}<0.05)$ between arm cycling and tonic contraction.

Figure 5. Group data (mean \pm SE) for CSPs $(n=10)$, recorded from the biceps brachii following TMS. Values from arm cycling (black bars) and tonic contractions (white bars) are shown for the three conditions. Results are expressed as absolute values (milliseconds). Asterisks denote a significant difference $(\mathrm{P}<0.05)$ between arm cycling and tonic contraction. 
A

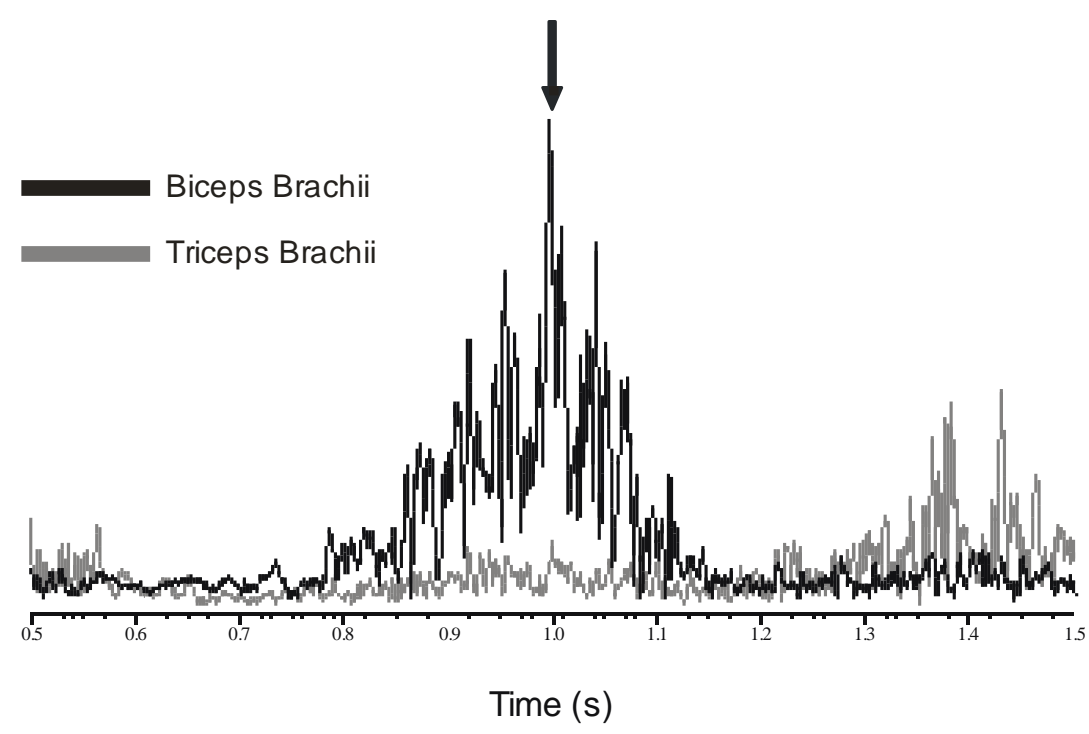

B

MEPs and CMEPs

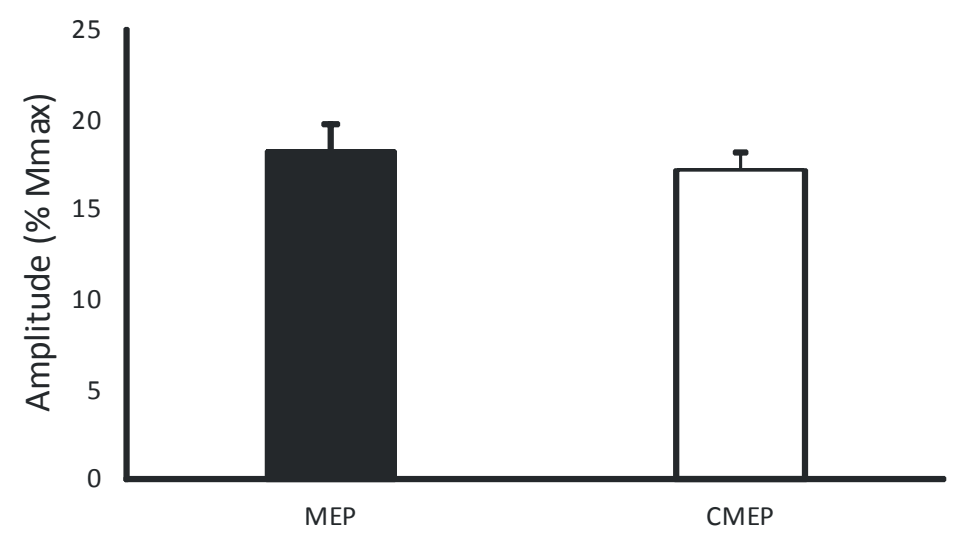

Figure 1 

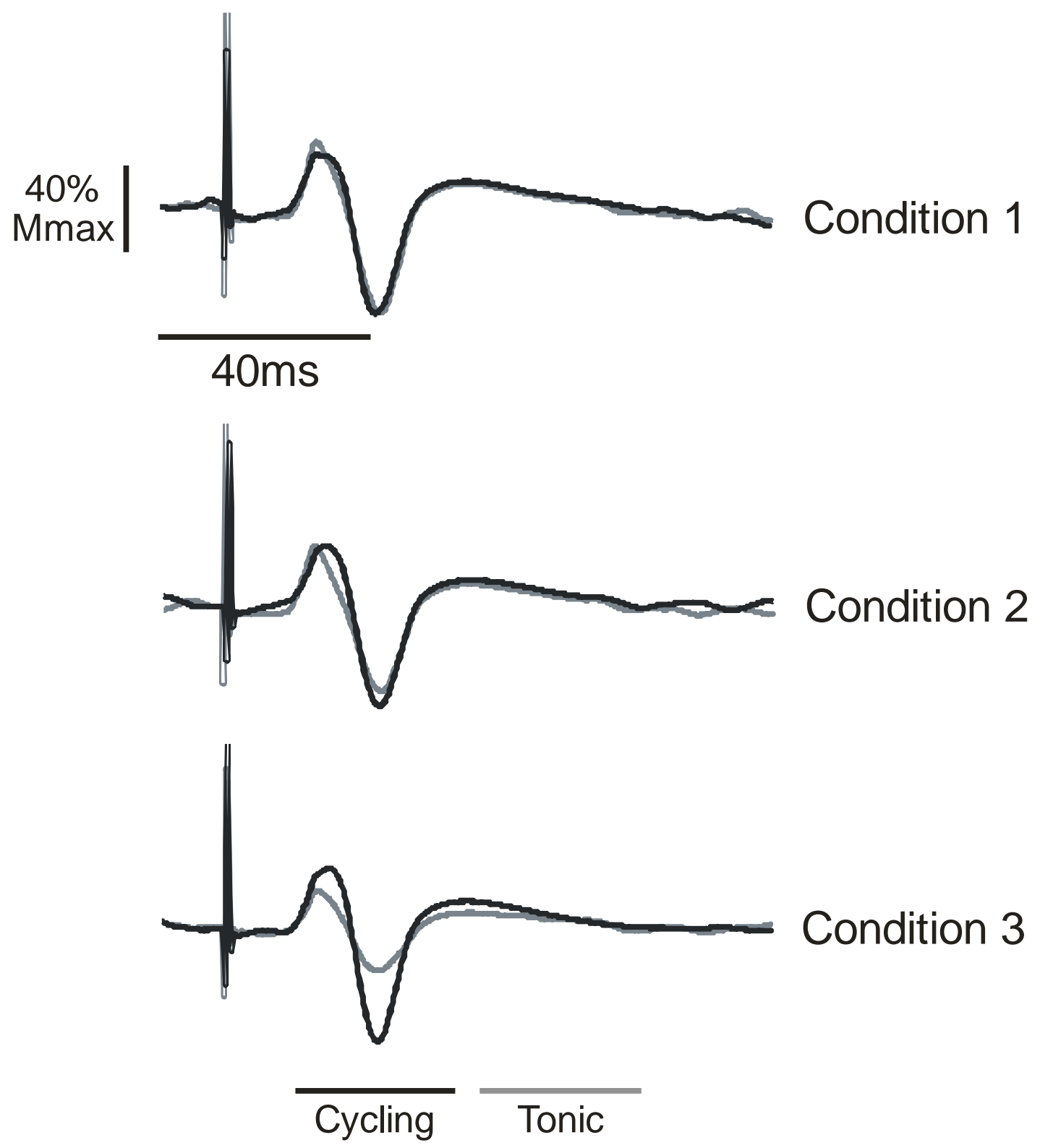

Figure 2 
A

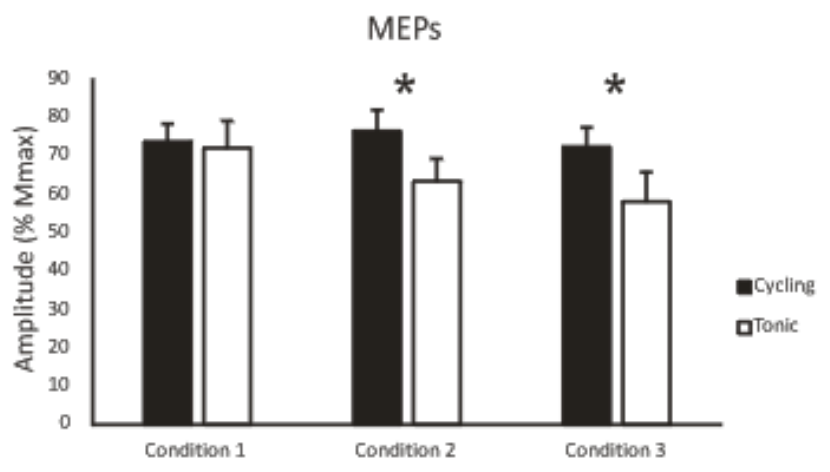

B Biceps Pre-Stimulus EMG

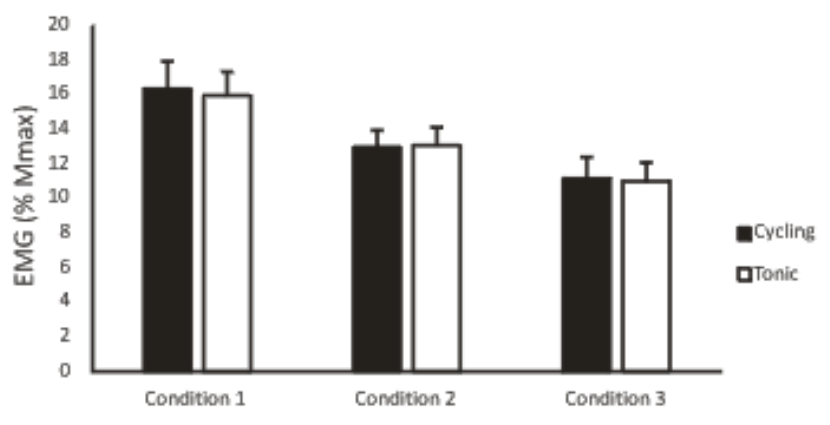

C Triceps Pre-Stimulus EMG

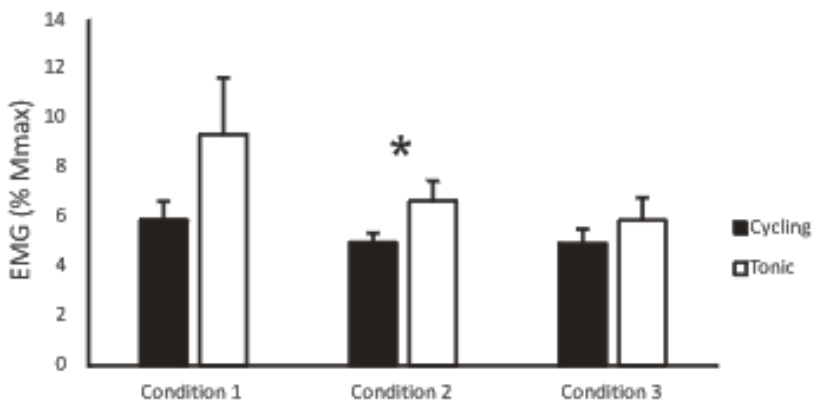

Figure 3 
A

CMEPS

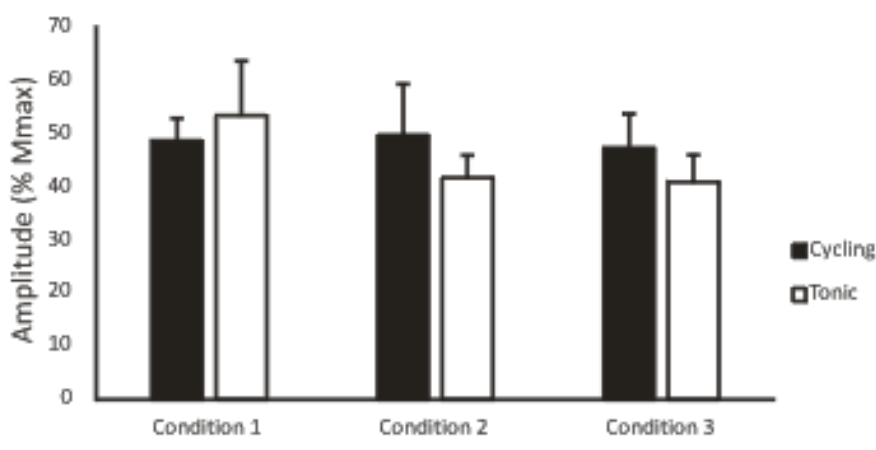

B

Biceps Pre-Stimulus EMG

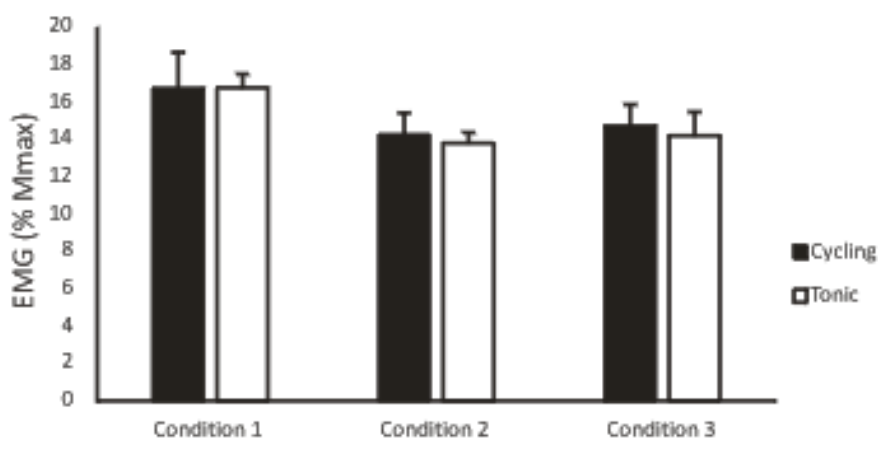

C Triceps Pre-Stimulus EMG

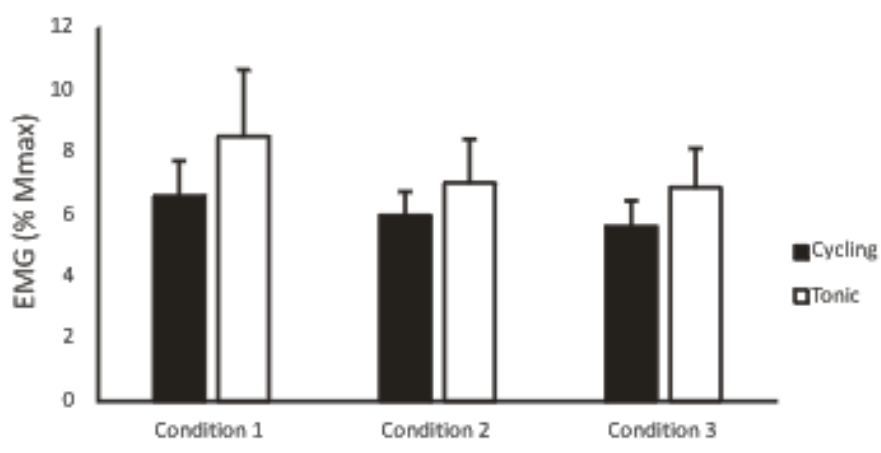

Figure 4 


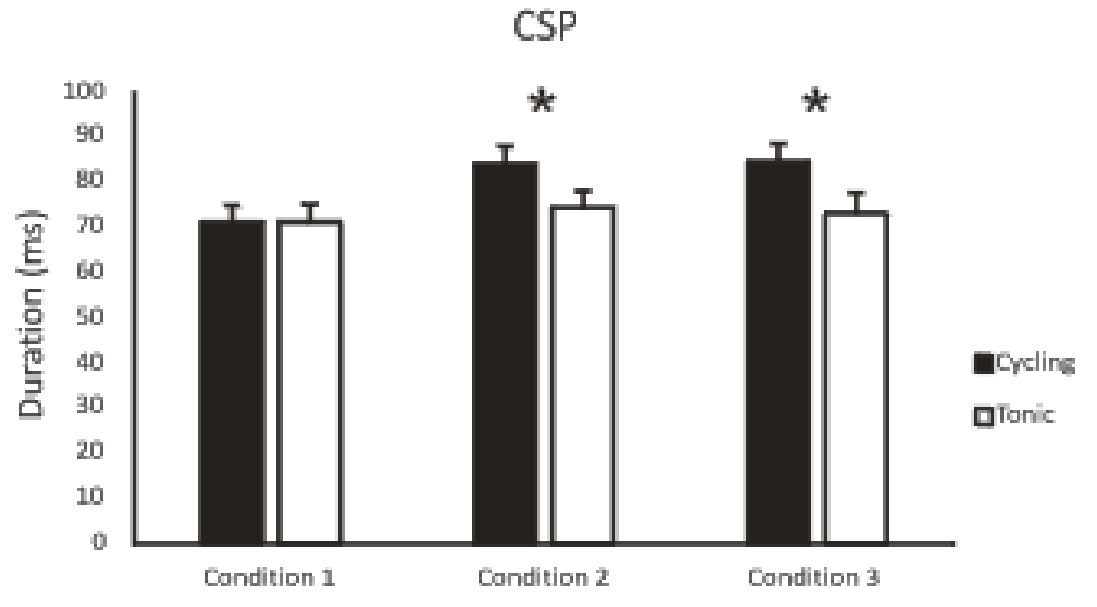

Figure 5 


\subsection{References}

Capaday C, Lavoie BA, Barbeau H, Schneider C, Bonnard M (1999). Studies on the corticospinal control of human walking. I. Responses to focal transcranial magnetic stimulation of the motor cortex. J Neurophysiol, 81: 129-139

Carroll TJ, Baldwin ER, Collins DF, Zehr EP (2006). Corticospinal excitability is lower during rhythmic arm movement than during tonic contraction. J Neurophysiol, 95: 914921

Copithorne DB, Forman D, Power K (2014). Premovement changes in corticospinal excitability of the biceps brachii are not different between arm cycling and an intensitymatched tonic contraction. Motor control

Crone C, Hultborn H, Jespersen B (1987). Reciprocal Ia inhibition between ankle flexors and extensors in man. J Physiol, 389:163-185

Forman D, Raj A, Button DC, Power K (2014). Corticospinal excitability of the biceps brachii is higher during arm cycling than an intensity-matched tonic contraction. $\mathrm{J}$ Neurophysiol, 112: 1142-1151

Gantchev N, Viallet F, Aurenty R, Massion J (1996). Impairment of posture-kinetic coordination during initiation of forward oriented stepping movements in parkinsonian patients. Electroencephalography and Clinical Neurophysiology/Electromyography and Motor Control, 101(2):110-120

Goto Y, Jono Y, Hatanaka R, Nomura Y, Tani Y, Chujo Y, Hiraoka K (2014). Different corticospinal control between discrete and rhythmic movement of the ankle. Frontiers in Human Neuroscience

Grillner S (1981). Control of locomotion in bipeds, tetrapods, and fish. In: Handbook of Physiology. The Nervous System. Motor Control. Bethesda, D: AM Physiol Soc, sect. 1, vol. 2: 1179-1236

Hiraoka K, Abe K. (2007) Cortical and spinal control of ankle joint muscles before and during gait initiation. Somatosensory and Motor Research, 24: 127-133

Jordan LM (1998). Initiation of locomotion in mammals. Ann NY Acad Sci, 860: 83-93 
Manning CD, Bawa P (2011). Heteronymous reflex connections in human upper limb muscles in response to stretch of forearm muscles. J Neurophysiol, 106:1489-1499

Nielsen J, Kagamihara Y, Crone C, Hultborn H (1992). Central facilitation of Ia inhibition during tonic ankle dorsiflexion revealed after blockade of peripheral feedback. Exp Brain Res, 88:651-656

Okada Y, Fukumoto T, Takatori K, Nagino K, Hiraoka K (2011). Abnormalities of the First Three Steps of Gait Initiation in Patients with Parkinson's Disease with Freezing of Gait. Parkinson's Disease, 2011

Orth M, Rothwell JC (2004). The cortical silent period: intrinsic variability and relation to the waveform of the transcranial magnetic stimulation pulse. Clin Neurophysiol, 115(5):1076-1082

Paulus W, Classen J, Cohen LG, Large CH, Di Lazzaro V, Nitsche M, Pascual-Leone A, Rosenow F, Rothwell JC, Ziemann U (2008). State of the art: Pharmacologic effects on cortical excitability measures tested by transcranial magnetic stimulation. Brain Stim, 1(3):151-163

Petersen NT, Taylor JL, Gandevia SC (2002). The effect of electrical stimulation of the corticospinal tract on motor units of human biceps brachii. J Physiol, 544: 277-284

Power KE, Copithorne DB. (2013) Increased corticospinal excitability prior to arm cycling is due to enhanced supraspinal but not spinal motoneurone excitability. Appl.

Physiol. Nutr. Metab., 38: 1154-1161

Pyndt HS, Laursen M, Nielsen JB (2003). Changes in Reciprocal Inhibition Across the Ankle Joint With Changes in External Load and Pedaling Rate During Bicycling. J Neurophysiol, 90:3168-3177

Pyndt HS, Nielsen JB (2003). Modulation of transmission in the corticospinal and group Ia afferent pathways to soleus motoneurones during bicycling. J Neurophysiol, 89: 304314

Rosin R, Topka H, Dichgans J (1997). Gait initiation in Parkinson's disease. Movement disorders, 12(5): 682-690 
Rossi S, Hallett M, Rossini PM, Pascual-Leone A (2009). Safety, ethical considerations, and application guidelines for the use of transcranial magnetic stimulation in clinical practice and research. Clin Neurophysiol, 120:2008-2039

Sidhu SK, Hoffmann BW, Cresswell AG, Carroll TJ. (2012) Corticospinal contributions to lower limb muscle activity during cycling in humans. J Neurophysiol, 107: 306-314

Takanaka R (1974). Reciprocal Ia inhibition During Voluntary Movements in Man. Exp Brain Res, 21:529-540

Zehr EP, Hundza SR, Vasudevan EV (2009). The quadrupedal nature of human bipedal locomotion. Exercise and sport sciences reviews, 37: 102-108

Zehr EP, Stein RB (1999). What function do reflexes serve during human locomotion? Progress in Neurobiology, 58: 185-205 